\title{
Improving Supply Chain Performance: Real-Time Demand Information and Flexible Deliveries
}

\author{
Kevin H. Shang \\ Fuqua School of Business, Duke University, Durham, North Carolina 27708, khshang@duke.edu \\ Sean X. Zhou \\ Systems Engineering \& Engineering Management, Chinese University of Hong Kong, \\ Shatin, Hong Kong, China, zhoux@se.cuhk.edu.hk \\ Geert-Jan van Houtum \\ School of Industrial Engineering, Eindhoven University of Technology, 5600 MB Eindhoven, The Netherlands, \\ g.j.v.houtum@tue.nl
}

\begin{abstract}
Tn some supply chains, materials are ordered periodically according to local information. This paper investiIgates how to improve the performance of such a supply chain. Specifically, we consider a serial inventory system in which each stage implements a local reorder interval policy; i.e., each stage orders up to a local basestock level according to a fixed-interval schedule. A fixed cost is incurred for placing an order. Two improvement strategies are considered: (1) expanding the information flow by acquiring real-time demand information and (2) accelerating the material flow via flexible deliveries. The first strategy leads to a reorder interval policy with full information; the second strategy leads to a reorder point policy with local information. Both policies have been studied in the literature. Thus, to assess the benefit of these strategies, we analyze the local reorder interval policy. We develop a bottom-up recursion to evaluate the system cost and provide a method to obtain the optimal policy. A numerical study shows the following: Increasing the flexibility of deliveries lowers costs more than does expanding information flow; the fixed order costs and the system lead times are key drivers that determine the effectiveness of these improvement strategies. In addition, we find that using optimal batch sizes in the reorder point policy and demand rate to infer reorder intervals may lead to significant cost inefficiency.
\end{abstract}

Key words: multi-echelon; periodic ordering; policy comparison; value of information; value of flexible deliveries

History: Received: May 6, 2008; accepted: July 28, 2009. Published online in Articles in Advance

November 13, 2009.

\section{Introduction}

In production/distribution systems, materials are often ordered and delivered in fixed time intervals. A location also usually orders according to its local inventory status. This inventory replenishment practice is commonly seen in a supply chain that comprises geographically dispersed entities, each with its own information systems. For example, $\mathrm{EMC}^{2}$, a leading manufacturer for database servers in the United States, implements this type of replenishment policy for some of its items. On fixed days of the week, $\mathrm{EMC}^{2}$ reviews inventory levels and places orders with its suppliers. After receiving the orders, the suppliers deliver materials to $\mathrm{EMC}^{2}$ via a third-party logistics truck if the requested items are available.
One reason for the widespread use of this replenishment practice is simplicity-it is just simpler to monitor local inventories and to plan personnel and labor for scheduled ordering and deliveries. In addition, this practice is particularly useful for a retailer who manages multiple products and needs to coordinate orders. However, it may create at least two operational inefficiencies. First, a location cannot order (so as to trigger a shipment) if it is not in one of its order periods. Second, real-time customer demand information cannot be transmitted immediately to upstream locations. This information delay may lead to inefficient order decisions at upstream stages.

We consider one such supply chain with fixed reorder intervals. To facilitate our discussion, we 
assume that the supply chain partners (who possibly belong to a single firm) share a common goal of optimizing systemwide performance, but they only have local information at the operational planning level. There is a central planner ${ }^{1}$ who determines all inventory control parameters (e.g., based on summary data in a spreadsheet), and each stage simply generates replenishment orders based on these control parameters and the local demand and inventory information. In this case, it is important to evaluate the current supply chain performance and to decide whether to invest to improve the supply chain. Here, we specifically consider two improvement strategiesexpanding the information flow by acquiring realtime demand information or accelerating the material flow by providing flexible deliveries. The question is, which strategy should be chosen and under what conditions?

This paper aims to answer this question by analyzing a periodic-review, serial inventory system with generally distributed one-period demands. As a base model, each stage implements a local reorder interval policy, referred to as the local $(s, T)$ policy. That is, a stage orders every $T$ periods according to its local inventory order position (=inventory on order + inventory on hand - local backorders). If the order position is less than the base-stock level $s$, the stage orders up to $s$. We assume that the reorder intervals follow integer-ratio relations; i.e., the reorder interval of an upstream stage is an integer-multiple of that of its immediate downstream stage. The replenishments are synchronized; that is, a downstream stage, whenever possible, places an order when its upstream stage receives a shipment. There is a fixed cost incurred for each order placed. Delivery lead times between stages are constant. There is a linear holding cost for each unit held at a stage and a linear penalty cost for each backorder that occurs at the most downstream stage. The objective is to minimize the average total supply chain cost per period. Note that the aforementioned inefficiencies appear in this base system: a stage cannot place an order in a period if the period is not an

\footnotetext{
${ }^{1}$ If the entire chain belongs to a single firm, the central planner can be the owner. For a chain composed of several independent firms, the central planner can be one of these firms, a team of them, or a third-party organization. We refer the reader to Shang et al. (2009, pp. 685-686) for practical examples.
}

order period, and the local inventory order position of a stage is updated by its immediate downstream orders, not by the demand received at the most downstream stage.

For the first improvement strategy, each stage can update its local inventory status according to the realtime demand information. It can be shown that the resulting system is equivalent to a system that implements an echelon $(s, T)$ policy. The echelon $(s, T)$ policy operates similarly to the local policy except that the echelon inventory order position (=inventory on order + inventory on hand + inventory at or in transit to all downstream stages - backorders at the most downstream stage) is monitored for each stage.

For the second improvement strategy, where a flexible delivery policy is implemented, a stage is allowed to place an order in any period. An upstream stage will fill and ship the order immediately, provided that the upstream stage has stock available. From this perspective, such a replenishment policy is more responsive to the downstream orders and hence can speed up the material flow. Because there is a fixed cost for placing an order, we assume that a stage will not place an order until its local inventory order position is lower than or equal to a threshold level $r$ and will order the least integer multiple of batch size $Q$ to raise the inventory order position above $r$. This replenishment scheme is essentially the same as the so-called local $(r, n Q)$ policies in the literature. For order coordination, we assume that the order batches satisfy integer-ratio relations: an upstream batch is an integer multiple of its immediate downstream one.

To answer our research question, we need to compare the optimal costs of the above mentioned three policies. Axsäter and Rosling (1993) showed that the local $(r, n Q)$ policy is a special case of the echelon $(r, n Q)$ policy (and that the local policy is therefore suboptimal). Chen (1998b) provided an algorithm to search for the optimal local reorder points when batch sizes are fixed. It is, however, not clear how to obtain the optimal local batch sizes. In Appendix B, we provide an approach to obtain these optimal batch sizes. For the echelon $(s, T)$ policy, van Houtum et al. (2007) provided a method to evaluate the echelon $(s, T)$ policy. They also constructed an algorithm for finding the optimal echelon base-stock levels when reorder intervals are fixed. Finally, a recent paper by Shang and 
Zhou (2009) provided an approach for obtaining the optimal reorder intervals for the echelon $(s, T)$ policy.

The main technical contribution of this paper is to analyze the local $(s, T)$ policy. We first show the dynamics of several key inventory variables under the local $(s, T)$ policy. These dynamics lead to a simple, bottom-up recursion that can evaluate a given local $(s, T)$ policy. More specifically, by converting the local inventory variables into echelon terms, we can evaluate the local policy as if it were an echelon $(s, T)$ policy with modified system lead times. This evaluation procedure can be used further to find the optimal local base-stock levels for given reorder intervals. Unlike the $(r, n Q)$ policy, the local $(s, T)$ policy is not a special case of the echelon $(s, T)$ policy. Nevertheless, we show that the optimal echelon $(s, T)$ policy always dominates the optimal local one. ${ }^{2}$ With this result, we provide a method to search for the optimal reorder intervals for the local $(s, T)$ policy.

These analytical results allow us to assess the value of the improvement strategies by comparing the aforementioned inventory policies in a numerical study. We find that the average (maximum) cost reduction when the system switches from the local $(s, T)$ policy to the local $(r, n Q)$ policy is $11.27 \%(28.32 \%)$, which is significantly larger than the $5.51 \%(16.67 \%)$ achieved when switching from the local $(s, T)$ policy to the echelon $(s, T)$ policy. This suggests that increasing the flexibility of delivery is more beneficial than acquiring realtime demand information. In addition, the difference between the benefits of both improvement strategies increases when demand becomes more variable. This result provides an important insight to avoid a management pitfall: when demand becomes more variable, it may not be effective to invest in IT systems in order to acquire real-time demand information; an agile logistics system is key to achieving supply chain efficiency. Our finding confirms the lessons from other papers, such as Cachon and Fisher (2000) and Gavirneni et al. (1999), in which the authors found that obtaining demand information may not be as valuable as improving physical system configurations.

\footnotetext{
${ }^{2}$ An echelon policy may not always dominate a local one. For example, Axsäter and Juntti (1996) showed that a local policy may dominate an echelon policy under some conditions for a twoechelon distribution system.
}

Here, we obtain a similar insight for a general-echelon model in which batch sizes and reorder intervals are determined endogenously (instead of exogenously as in the above two papers). Of course, our conclusion does not consider other nonmodeled factors, such as implementation costs, labor costs, and easiness of execution, which tend to favor the $(s, T)$ policy. A manager who has to make a choice between the two improvement policies also has to take these nonmodeled factors into account.

We further use our model to examine the issue of value of demand information (VOI). The VOI in our model is equivalent to the cost reduction by switching from the local policy to the echelon policy. In a numerical study, we find that there is no significant benefit achieved by switching from the local $(r, n Q)$ policy to the echelon $(r, n Q)$ policy. This observation suggests that adding demand information to a system with flexible deliveries provides little value. Interestingly, Chen (1998b) conducted a similar study with fixed, exogenous batch sizes and reported a slightly higher average VOI. For the $(s, T)$ policies, the VOI is higher when the fixed costs are larger and lead times are shorter. This implies that the demand information is most beneficial when the supply chain has long reorder intervals (higher fixed costs lead to longer reorder intervals) and short lead times. In $\S 4$, we provide intuitive explanations for the above observations.

Finally, we investigate whether the reorder intervals can be inferred by the optimal batch sizes and the demand rate. The logic behind this question is that the reorder intervals and batch sizes are equivalent in the deterministic demand model. Thus it is plausible to conjecture that effective reorder intervals may be inferred by the optimal batch sizes in the stochastic demand model. Interestingly, our numerical study suggests that the inferred reorder intervals are seldom optimal and may lead to significant cost inefficiency when the system only has local information.

\section{Literature Review}

Our paper is related to the literature of inventory control in multi-echelon systems with $(r, n Q)$ and $(s, T)$ policies. The local and echelon $(r, n Q)$ policies have been extensively studied in the literature. Available results include policy evaluation, optimization, and approximations. Noteworthy examples include 
Axsäter (1993), Cachon (2001a), De Bodt and Graves (1985), Chen (1998b, 2000), Chen and Zheng (1998), Shang (2008), and Shang and Song (2007). We refer the reader to Axsäter (2003), Chen (1998a), and SimchiLevi and Zhao (2007) for a review. The literature on the $(s, T)$ policy is relatively sparse. Naddor (1975) studied the $(s, T)$ policy for single- and multi-item systems. Cachon (1999) studied the reorder-interval policy in a distribution system. He showed that the supplier's demand variance will decline as the retailers' reorder interval becomes longer. Graves (1996) provided a new approach to evaluate the cost for distribution systems under the so-called virtual allocation rule. Van Houtum et al. (2007) studied a serial model and showed that the echelon $(s, T)$ policies are optimal when the reorder intervals are fixed. They also provided an algorithm to obtain the optimal base-stock levels. Chao and Zhou (2009) extended these results to batch-ordering systems. Feng and Rao (2007) considered a two-stage system with echelon $(s, T)$ policies. They provided a heuristic for the policy parameters. Recently, Shang and Zhou (2009) provided a heuristic and an approach for obtaining the exact optimal $(s, T)$ policy when fixed order costs are present.

Our paper is also related to the literature on comparisons of inventory policies. For single-stage systems, Hadley and Whitin (1963) compared the $(r, n Q)$ and $(s, T)$ policies and showed that the $(r, n Q)$ policy is superior to the $(s, T)$ policy. Rao (2003) studied the $(s, T)$ policy under the continuous-time model and constructed a worst-case cost bound for the optimal $(s, T)$ policy based on the optimality result of the $(r, Q)$ policy. For multilocation models, Cachon (2001b) compared three policies, i.e., $(Q, S),(Q, S \mid T)$, and $(S, T)$ policies, in a multiretailer system. The $(Q, S)$ and $(S, T)$ policies are similar to our $(r, n Q)$ and $(s, T)$ policies, respectively. His study suggested that the policies with fixed replenishment intervals may lead to significant inefficiency. Recently, Gürbüz et al. (2007) also studied a multiretailer model. They proposed a new inventory policy and compared it with the three existing ones. They identified the situations under which each policy would perform effectively.

Finally, our paper adds to the literature on assessing the VOI. The literature in this subject is extensive. Most models assume either two-echelon systems or that batch sizes/reorder intervals are fixed (for example, see Aviv and Federgruen 1998, Cachon and Fisher 2000, Chen 1998b, Gavirneni 2002, Gavirneni et al. 1999, Graves 1999, Lee et al. 2000, and Simchi-Levi and Zhao 2004). Simchi-Levi and Zhao (2004) studied a two-stage serial system where a capacitated manufacturer supplies a retailer. Both stages implement an $(s, T)$ policy, but the retailer has a longer reorder interval than the manufacturer. They found that if the retailer shares the demand information between order periods, the system cost can be reduced significantly.

The remainder of this paper is organized as follows: $\$ 2$ introduces the base model and the local $(s, T)$ policy. We also discuss the two improvement strategies and the resulting inventory policies. Section 3 analyzes the local $(s, T)$ policy, and we show how to evaluate and optimize the local $(s, T)$ policy. Section 4 conducts a numerical study to compare these inventory policies, with an emphasis on the effectiveness of the improvement strategies. We also identify the conditions under which each of the strategies is most beneficial. Section 5 concludes. Appendix A presents the proofs. Appendix B provides an approach for obtaining optimal batch sizes for the local $(r, n Q)$ policy.

\section{The Base System and Two Improvement Strategies}

We consider a centralized-control, periodic-review serial inventory system with $N$ stages $(N \in \mathbb{N}$, the set of positive integers, $N \geq 2$ ). Customer demand occurs at stage 1 . Stage 1 is replenished by stage 2 , stage 2 by stage 3 , etc., and stage $N$ by an outside source with ample supplies. Time is divided into periods of length one, and the periods are numbered $0,1,2, \ldots$ Let $[t, t+\tau)$ and $[t, t+\tau]$ denote the time interval over periods $t, t+1, \ldots, t+\tau-1$ and periods $t, t+1, \ldots, t+\tau$, respectively. Demands are independently and identically distributed between periods. Let $\mu(>0)$ denote the mean of demand in a period and $D[t, t+\tau)$ and $D[t, t+\tau]$ denote the cumulative demand over time in $[t, t+\tau)$ and in $[t, t+\tau]$. We use $D[\tau)$ and $D[\tau]$ to represent the demand in $\tau$ and in $\tau+1$ periods, respectively, if the time index $t$ is omitted. There are constant lead times $L_{j} \in \mathbb{N}$ between stages $j+1$ and $j$. At the end of each period, the echelon holding cost $h_{j}(\geq 0)$ is incurred for each unit of 
inventory held in echelon $j$, and the backorder cost $b$ $(>0)$ is incurred for each unit of backorders at stage 1. Denote $h_{[i, j]}=\sum_{k=i}^{j} h_{k}(1 \leq i \leq j \leq N)$, so the local holding cost for stage $j$ is $h_{[j, N]}$.

The base model operates under the local $(s, T)$ policy. Under this policy, stage $j$ views stage $j-1$ 's order as its demand and orders every $T_{j}$ periods. At the beginning of an order period, referred to as an order epoch, stage $j$ reviews its local inventory order position (=outstanding orders + on-hand inventory - stage $j$ 's backorders) and orders up to the local base-stock level $s_{j}$. There is a fixed cost $k_{j}(>0)$ incurred for placing an order. The ordering times between stages are synchronized. That is, stage $j$ orders, whenever possible, when stage $j+1$ receives a shipment. This rule was first introduced by Graves (1996), and we hereafter term this rule the synchronized replenishment rule. (It can be shown that the synchronized replenishment rule is the best for the local $(s, T)$ policy. A proof is available from the authors upon request.) In addition, the reorder intervals satisfy integer-ratio relations; i.e., $T_{j}=n_{j} T_{j-1}, n_{j} \in \mathbb{N}$, for $j=2, \ldots, N$.

We assume that the replenishment activities in a period occur at the beginning of the period. At stage $j>1$, they occur in the following sequence: (1) an order, if any, from stage $j-1$ is received; (2) an order is placed with stage $j+1$ if the period is in stage $j$ 's order period; (3) a shipment, if any, is received from stage $j+1$; and (4) a shipment is sent to stage $j-1$ if the period is in stage $j-1$ 's order period. For stage 1 , order placement occurs at the beginning of stage 1's order periods, whereas customer demand arrives during a period. We assume that the stages perform these events sequentially, from stage 1 , stage 2 , etc., until stage $N$. Costs are evaluated at the end of a period. The objective is to minimize the average total cost per period.

We use a two-stage example (see Figure 1) to illustrate the system dynamics under the local $(s, T)$ policy. In this example, $T_{1}=2, T_{2}=4, L_{1}=1$, and $L_{2}=1$. Define

$$
\begin{aligned}
I O P_{j}^{\prime}-(t)= & \text { local inventory order position after } \\
& \text { receiving downstream orders at the } \\
& \text { beginning of period } t, \\
I O P_{j}^{\prime}(t)= & \text { local inventory order position after } \\
& \text { placing an order at the beginning } \\
& \text { of period } t .
\end{aligned}
$$

Figure 1 The Dynamics of $I O P_{j}^{\prime}$ Under the Local $(s, T)$ Policy
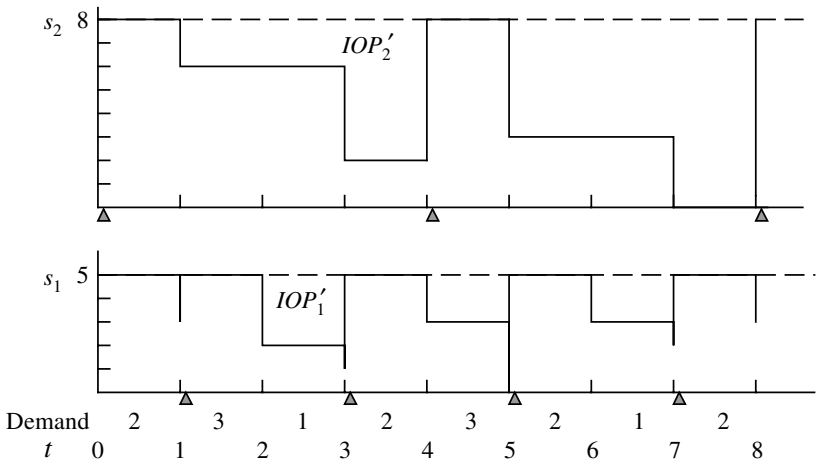

Note. The triangles represent the order epochs.

We assume that the system starts with $I O P_{j}^{\prime}(0)=s_{j}$, for all $j$. In this example, $s_{1}=5$ and $s_{2}=8$. Stage 2 orders at $t=0,4,8, \ldots$. These orders will arrive at $t=1,5,9, \ldots$ Based on the synchronized replenishment rule, stage 1 should order at $t=1,3,5, \ldots$. Let us focus on $I O P_{2}^{\prime}$ at stage 2. At $t=1$ and $t=3$, stage 1 places an order. These orders are the demand for stage 2 , and thus $I O P_{2}^{\prime}$ decreases. At $t=4$, $I_{O P}^{\prime-}(4)=2$, so stage 2 places an order of six units. These six units are the customer demand that occurs in $[0,3)$. Note that stage 1 receives two units of customer demand in $[3,4)$. Because stage 1 does not order at $t=4$, stage 2 does not consider these two units of demand when it places an order. We will analyze the local $(s, T)$ policy in detail in the next section. For now, let us turn to each of the improvement strategies.

\subsection{Real-Time Demand Information}

For the first strategy, the supply chain can invest in IT systems to make the demand information available to all stages. In such a case, a stage can update its local inventory status according to the real-time demand information. Following Shang et al. (2009), it can be shown that the resulting system is equivalent to a system in which stage $j$ implements an echelon $(s, T)$ policy with the echelon base-stock level

$$
S_{j}^{e}=\sum_{i=1}^{j} s_{i}
$$

and the reorder interval $T_{j}, j=1, \ldots, N$. Notice that the resulting echelon $(s, T)$ policy also follows the synchronized replenishment rule. The echelon $(s, T)$ policy is operated similarly as the local $(s, T)$ policy 
except that the echelon inventory order position is monitored. More specifically, let

$$
\begin{aligned}
I O P_{j}^{-}(t)= & \text { echelon inventory order position after } \\
& \text { receiving customer demand } D[t-1, t) \\
& \text { at the beginning of period } t \text { and before } \\
& \text { ordering }=\sum_{i=1}^{j} I O P_{i}^{\prime}-(t), \\
I O P_{j}(t)= & \text { echelon inventory order position after } \\
& \text { ordering at the beginning of period } \\
& t=\sum_{i=1}^{j} I O P_{i}^{\prime}(t) .
\end{aligned}
$$

Under the echelon $(s, T)$ policy, each stage $j$ orders in every $T_{j}$ periods. At an order epoch $t$, if $I O P_{j}^{-}(t)$ is less than $S_{j}^{e}$, the stage orders up to $S_{j}^{e}$ so that $I O P_{j}(t)=S_{j}^{e}$.

The echelon $(s, T)$ policy has been studied recently. We summarize several key results which will be used in the subsequent sections. Define $\mathbf{T}_{\mathbf{j}}=\left(T_{1}, \ldots, T_{j}\right)$, $j=1, \ldots, N, \mathbf{T}=\mathbf{T}_{\mathbf{N}}$, and $x^{-}=\max \{0,-x\}$. For given echelon base-stock levels $\left(S_{1}^{e}, \ldots, S_{N}^{e}\right)$, the average inventory holding and backorder cost can be evaluated by the following recursion (van Houtum et al. 2007 and Chao and Zhou 2009): Define

$$
\begin{aligned}
G_{1}^{e}\left(y, T_{1}\right)=\frac{1}{T_{1}}( & \sum_{l=0}^{T_{1}-1} \mathrm{E}\left[h_{1}\left(y-D\left[L_{1}+l\right]\right)\right. \\
& \left.\left.+\left(b+h_{[1, N]}\right)\left(y-D\left[L_{1}+l\right]\right)^{-}\right]\right) .
\end{aligned}
$$

For $j=2, \ldots, N$,

$$
\begin{aligned}
& G_{j}^{e}\left(y, \mathbf{T}_{\mathbf{j}}\right) \\
& =\frac{1}{T_{j}} \sum_{l=0}^{T_{j}-1} \mathrm{E}\left[h_{j}\left(y-D\left[L_{j}+l\right]\right)\right. \\
& \left.+G_{j-1}^{e}\left(\min \left\{S_{j-1}^{e},\left(y-D\left[L_{j}+\left\lfloor\frac{l}{T_{j-1}}\right\rfloor T_{j-1}\right)\right)\right\}, \mathbf{T}_{\mathbf{j}-1}\right)\right] .
\end{aligned}
$$

Here, $G_{j}^{e}\left(y, \mathbf{T}_{\mathbf{j}}\right)$ represents the average inventory holding and backorder costs of echelon $j$ when stage $j+1$ has infinite stock (so that orders of stage $j$ are always immediately fulfilled) and stages $1, \ldots, j-1, j$ follow an echelon $(s, T)$ policy with base-stock levels $S_{1}^{e}, \ldots, S_{j-1}^{e}, y$.

For the average fixed cost, note that $k_{j}$ is incurred only when stage $j$ places an order. Thus the probability of ordering at each stage $j$ 's order epoch $t$ is $\mathrm{P}\left(I O P_{j}^{-}(t)<S_{j}^{e}\right)$. Under the echelon $(s, T)$ policy, $I O P_{j}\left(t-T_{j}\right)=S_{j}^{e}$ and $I O P_{j}^{-}(t)=I O P_{j}\left(t-T_{j}\right)-D\left[T_{j}\right)$. Thus the probability of ordering is equal to $p_{j}=$ $\mathrm{P}\left(D\left[T_{j}\right)>0\right)$. With this, the average fixed cost is equal to $\sum_{i=1}^{N}\left(k_{i} p_{i} / T_{i}\right)$. Consequently, the average total cost per period is $C^{e}\left(\mathbf{S}^{\mathbf{e}}, \mathbf{T}\right)=\sum_{j=1}^{N}\left(k_{j} p_{j} / T_{j}\right)+$ $G_{N}^{e}\left(S_{N}^{e}, \mathbf{T}_{\mathbf{N}}\right)$.

For fixed $\mathbf{T}$, the average fixed cost term is a constant. Thus minimizing $C^{e}\left(\mathbf{S}^{\mathbf{e}}, \mathbf{T}\right)$ is equivalent to minimizing $G_{N}^{e}\left(S_{N}^{e}, \mathbf{T}_{\mathbf{N}}\right)$. A solution that minimizes $G_{N}^{e}\left(S_{N}^{e}, \mathbf{T}_{\mathbf{N}}\right)$ can be obtained by minimizing (1) and (2) recursively. More specifically, let $S_{1}^{e}\left(\mathbf{T}_{\mathbf{1}}\right)=\operatorname{argmin}_{y} G_{1}^{e}\left(y, \mathbf{T}_{1}\right)$. For $j=$ $2, \ldots, N$, suppose that $S_{j-1}^{e}\left(\mathbf{T}_{\mathbf{j}-1}\right)$ is known. Substitute $S_{j-1}^{e}\left(\mathbf{T}_{\mathbf{j}-1}\right)$ for $S_{j-1}^{e}$ in (2) and let $S_{j}^{e}\left(\mathbf{T}_{\mathbf{j}}\right)$ be the minimizer of the resulting $G_{j}^{e}\left(y, \mathbf{T}_{\mathbf{j}}\right)$ function; i.e., $S_{j}^{e}\left(\mathbf{T}_{\mathbf{j}}\right)=$ $\operatorname{argmin}_{y} G_{j}^{e}\left(y, \mathbf{T}_{\mathbf{j}}\right)$. Then $\mathbf{S}^{\mathbf{e}}(\mathbf{T})=\left(S_{1}^{e}\left(T_{1}\right), \ldots, S_{N}^{e}\left(\mathbf{T}_{\mathbf{N}}\right)\right)$ is the optimal echelon base-stock vector.

Finally, to find the optimal reorder intervals for the echelon policy, one needs to solve the following problem:

$$
\begin{array}{ll}
\min _{\mathbf{T}} & C^{e}(\mathbf{T})=C^{e}\left(\mathbf{S}^{\mathbf{e}}(\mathbf{T}), \mathbf{T}\right), \\
\text { s.t. } & T_{j}=n_{j} T_{j-1}, \quad j=2, \ldots, N .
\end{array}
$$

We refer the reader to Shang and Zhou (2009) for an algorithm.

\subsection{Flexible Deliveries}

For the second strategy in which a flexible delivery policy is implemented, stage $j$ is allowed to place an order in any period, and stage $j+1$ will immediately ship the order to stage $j$ if stage $j+1$ has sufficient stock. Because there is a fixed cost associated with each order placed, we assume that stage $j$ will not place an order until its inventory order position is lower than or equal to a threshold level $r_{j}$ and will order the least integer multiple of batch size $Q_{j}$ to raise the inventory order position above $r_{j}$. In other words, this policy is essentially the same as the local $(r, n Q)$ policy. More specifically, for any period $t$, if $I O P_{j}^{\prime}-(t) \leq r_{j}$, stage $j$ places an order of $n Q_{j}, n \in \mathbb{N}$, such that $I O P_{j}^{\prime}(t) \in$ $\left\{r_{j}+1, r_{j}+2, \ldots, r_{j}+Q_{j}\right\}$. Here, $k_{j}$ is incurred once for placing an order that may include several batches. We assume that the order batches satisfy integer-ratio constraints: $Q_{j}=q_{j} Q_{j-1}, q_{j} \in \mathbb{N}, j=2, \ldots, N$. Also, the reorder point $r_{j}$ is assumed to be an integer multiple of its downstream batch size $Q_{j-1}$ (Chen 1998b). 
Note that under this policy, the upstream stage's local inventory order position is only updated by the downstream stage's orders, not by the real-time customer demand at stage 1.

Axsäter and Rosling (1993) showed that for any local $(r, n Q)$ policy, there exists an equivalent echelon $(r, n Q)$ policy. Shang and Zhou (2009) developed a scheme to evaluate an echelon $(r, n Q)$ policy with fixed order costs incurred for each order placed. We can use these results to evaluate a local $(r, n Q)$ policy. Specifically, we first convert a local $(r, n Q)$ policy to the corresponding echelon policy and then apply Shang and Zhou's recursion to evaluate the cost. With fixed batch sizes, Chen (1998b) developed an approach to find the best reorder points for the local $(r, n Q)$ policy. It is, however, not known how to find the optimal batch sizes for the local $(r, n Q)$ policy in the literature. In Appendix B, we provide an approach to obtain the bounds for the optimal local batch sizes. The optimal solution then can be found by a search over all feasible solutions.

\section{The Local $(s, T)$ Policy: Evaluation and Optimization}

This section analyzes the local $(s, T)$ policy, aiming to answer several questions. First, how does one evaluate a local $(s, T)$ policy? Second, how does one obtain the optimal local policy parameters? Third, what is the relationship between the local and echelon $(s, T)$ policies? Is the local policy a special case of the echelon one? If not, does the echelon policy dominate the local one?

We first provide a method to evaluate the total cost. It is simpler to evaluate the cost from the echelon perspective. Recall the definition of $\operatorname{IOP}_{j}(t)$. We further define

$I L_{j}^{-}(t)=$ net echelon inventory level for stage $j$ at the beginning of period $t$ (after order arrival),

$I L_{j}(t)=$ net echelon inventory level for stage $j$ at the end of period $t$,

$I P_{j}(t)=$ echelon inventory in-transit position for stage $j$ at the beginning of period $t$ after ordering.

Note that the difference between $I O P_{j}(t)$ and $I P_{j}(t)$ is the outstanding orders for stage $j$ that are not yet filled by stage $j+1$. Also, the total cost is determined by the $I L_{j}(t)$ values.

Consider the dynamics of the echelon inventory variables under the local $(s, T)$ policies. Suppose that stage $N$ places an order at period $t$. Let the resulting echelon inventory order position be $I O P_{N}(t)$. Because stage $N$ has ample supply, $I P_{N}(t)=I O P_{N}(t)$. This order will arrive at stage $N$ at period $t+L_{N}$. Because there will be no other order periods until period $t+T_{N}, I P_{N}(t)$ will determine both $I L_{N}^{-}\left(t+L_{N}+\tau\right)$ and $I L_{N}\left(t+L_{N}+\tau\right)$ for $\tau=0, \ldots, T_{N}-1$. That is,

$$
I L_{N}^{-}\left(t+L_{N}+\tau\right)=I P_{N}(t)-D\left[t, t+L_{N}+\tau\right),
$$

and

$$
I L_{N}\left(t+L_{N}+\tau\right)=I P_{N}(t)-D\left[t, t+L_{N}+\tau\right] .
$$

Now consider stage $j=N-1, N-2, \ldots, 1$ sequentially. Let $\mathbb{M}_{b}(a)$ be the operator that returns the remainder of $a$ divided by $b$, and $a, b \in \mathbb{N}$ and $L_{[i, j]}=$ $\sum_{k=i}^{j} L_{k}$ the total lead times between stage $i$ and $j+1$. According to the synchronized replenishment rule, stage $j$ will order in periods $t+L_{[j+1, N]}+\left\lfloor\tau / T_{j}\right\rfloor T_{j}$, for $\tau=0, \ldots, T_{N}-1$. The resulting stage $j$ 's echelon inventory order position and the stage $j+1$ 's net echelon inventory level jointly determine stage $j$ 's echelon intransit inventory position. That is, for $\tau=0, \ldots, T_{N}-1$,

$$
\begin{array}{r}
I P_{j}\left(t+L_{[j+1, N]}+\left\lfloor\frac{\tau}{T_{j}}\right\rfloor T_{j}\right) \\
=\min \left\{I O P_{j}\left(t+L_{[j+1, N]}+\left\lfloor\frac{\tau}{T_{j}}\right\rfloor T_{j}\right),\right. \\
\left.I L_{j+1}^{-}\left(t+L_{[j+1, N]}+\left\lfloor\frac{\tau}{T_{j}}\right\rfloor T_{j}\right)\right\} .
\end{array}
$$

Each $I P_{j}$ will further determine $I L_{j}^{-}$and $I L_{j}$ :

$$
\begin{aligned}
& I L_{j}^{-}\left(t+L_{[j, N]}+\tau\right) \\
& =I P_{j}\left(t+L_{[j+1, N]}+\left\lfloor\frac{\tau}{T_{j}}\right\rfloor T_{j}\right)-D\left[t+L_{[j+1, N]}+\left\lfloor\frac{\tau}{T_{j}}\right\rfloor T_{j},\right. \\
& \left.t+L_{[j, N]}+\left\lfloor\frac{\tau}{T_{j}}\right\rfloor T_{j}+\mathbb{M}_{T_{j}}(\tau)\right), \\
& I L_{j}\left(t+L_{[j, N]}+\tau\right) \\
& =I P_{j}\left(t+L_{[j+1, N]}+\left\lfloor\frac{\tau}{T_{j}}\right\rfloor T_{j}\right)-D\left[t+L_{[j+1, N]}+\left\lfloor\frac{\tau}{T_{j}}\right\rfloor T_{j},\right. \\
& \left.t+L_{[j, N]}+\left\lfloor\frac{\tau}{T_{j}}\right\rfloor T_{j}+\mathbb{M}_{T_{j}}(\tau)\right\rfloor .
\end{aligned}
$$


Consequently, if we can determine $\operatorname{IOP}_{j}(\cdot)$ in (3), we are able to determine $I L_{j}^{-}(\cdot)$ and $I L_{j}(\cdot)$ from the recursion (3)-(5). Our next task is to determine these $\operatorname{IOP}_{j}(\cdot)$ under the local $(s, T)$ policy.

Recall that the local base-stock levels are given by $\left(s_{1}, \ldots, s_{N}\right)$, and define

$$
S_{j}=\sum_{i=1}^{j} s_{i} \quad \text { for } j=1, \ldots, N .
$$

We call $S_{j}$ the induced echelon base-stock level, as it is determined by the local base-stock levels. Because the system starts with $\operatorname{IOP}_{j}^{\prime}(0)=s_{j}, \operatorname{IOP}_{j}(0)=$ $\sum_{i=1}^{j} \operatorname{IOP}_{i}^{\prime}(0)=\sum_{i=1}^{j} s_{i}=S_{j}$. As we illustrated in $\S 2$, under the local $(s, T)$ policy, stage $j$ will order a quantity equal to the total orders received from stage $j-1$ since the last order epoch. However, demand may have occurred at stage 1 , of which stage $j$ was not aware when placing the order. Thus the echelon inventory order position $I O P_{j}(t)$ may not be $S_{j}$ under the local $(s, T)$ policy.

To further explain this, let us first consider a three-stage example with $T_{1}=2, T_{2}=T_{3}=4, L_{1}=L_{2}=$ $L_{3}=1$ (see Figure 2). If, say, stage 3 orders at $t=$ $3,7,11, \ldots$, then stage 2 will order at $t=0,4,8,12, \ldots$ and stage 1 will order at $t=1,3,5,7, \ldots$ because of the synchronized replenishment rule. In this example, $I O P_{1}^{\prime}(0)=s_{1}=5, I O P_{2}^{\prime}(0)=s_{2}=8$, and $I O P_{3}^{\prime}(0)=s_{3}=$ 14 , so $I O P_{1}(0)=5, I O P_{2}(0)=13$, and $I O P_{3}(0)=27$. Figure 2(a) shows $I O P_{j}^{\prime}(t)$ for the local $(s, T)$ policy for a given demand sample path; Figure $2(b)$ is the corresponding $\operatorname{IOP}_{j}(t)$ resulting from the local $(s, T)$ policy.

First, let us consider the local policy at stage 2 at $t=4$ (Figure 2(a)). At this order epoch, stage 2 orders four units to bring its $\operatorname{IOP}_{2}^{\prime}(4)$ to $s_{2}$. These four units come from the two orders placed by stage 1 at $t=$ 1 and $t=3$, which account for the total demand in the time interval $[0,3)$. Note that when stage 2 places an order at $t=4$, stage 1 actually has received two units of demand in $[3,4)$. However, because stage 1 cannot order until $t=5$, stage 2 does not order two additional units for the demand in $[3,4)$. Thus the echelon inventory order position $\operatorname{IOP}_{2}(4)=S_{2}-2=11$, which is shown in Figure 2(b). It is this information delay that causes the inefficiency of the system.

A similar situation applies for stage 3 at $t=7$. At this order epoch, stage 3 places an order of four units
Figure 2 (a) The Dynamics of $I O P_{j}^{\prime}(t)$ Under the Local $(s, T)$ Policy, Where the Triangles Represent Order Epochs. (b) The Corresponding Echelon Inventory Order Position $10 P_{j}(t)$
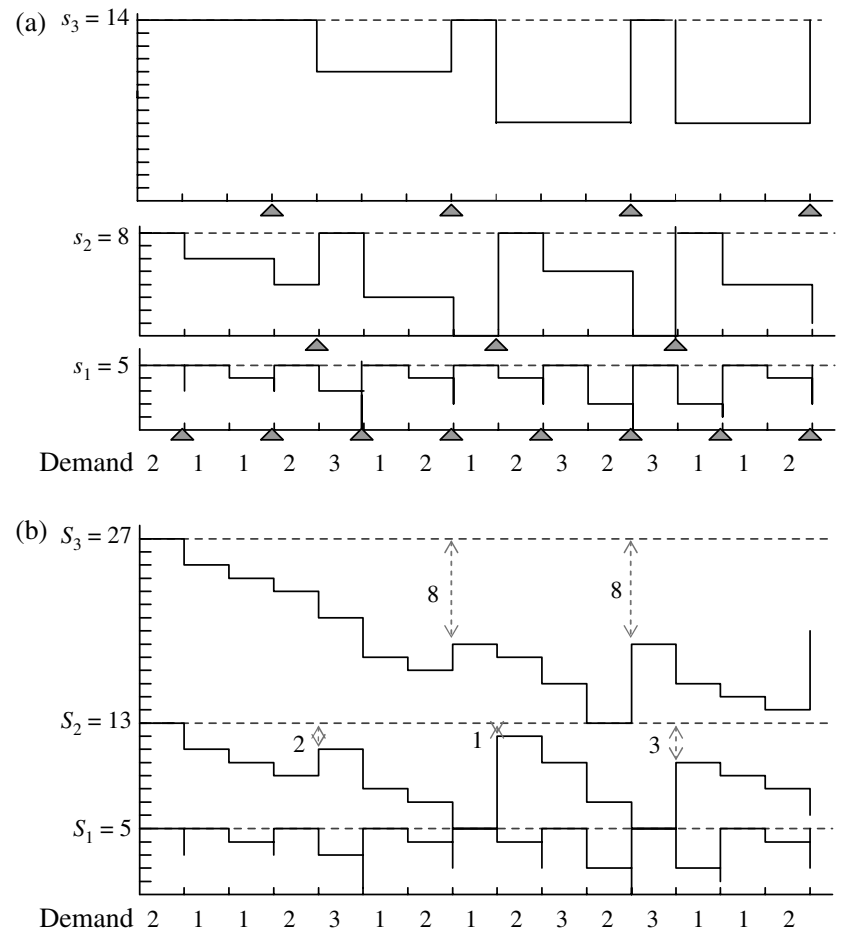

to account for stage 2's order at $t=4$. These four units, again, are to account for the demand in $[0,3)$. Because of the order delay, stage 3 does not learn of the demand in $[3,7)$, which is equal to $2+3+1+2=$ 8 units. Thus the echelon inventory order position for stage 3 at the order epoch $t=7$ is $I_{3} P_{3}(7)=S_{3}-$ $D[3,7)=27-8=19$.

We generalize the above observation in the following proposition. Define a notation, " $\ominus$," where

$$
(a \ominus b)=a n-b, \quad a, b \in \mathbb{N},
$$

and $n$ is the smallest positive integer such that $a n \geq b$.

Proposition 1. For local $(s, T)$ policies, the resulting echelon inventory order position $\mathrm{IOP}_{j}(t)$ at an order epoch $t$ at stage $j$ is $S_{j}-D\left[t-\sum_{i=2}^{j}\left(T_{i-1} \ominus L_{i}\right), t\right]$.

We make two remarks here. First, the random variable $D\left[t-\sum_{i=2}^{j}\left(T_{i-1}-L_{i}\right), t\right]$ is the amount of demand occurred at stage 1 but not yet learned by stage $j$ when stage $j$ places an order under the local $(s, T)$ policy. Thus it can be viewed as the amount of delayed demand information. For the special case 
where $T_{j}=1$ for all $j$, this term reduces to zero, which implies that no demand information is delayed under the classic local base-stock policy (so the local basestock policy is equivalent to the echelon base-stock policy, and vice versa).

Second, recall that the echelon inventory order position $\operatorname{IOP}_{j}(t)$ resulting from the echelon $(s, T)$ policy is always $S_{j}^{e}$. Here, $I O P_{j}(t)$ for the local $(s, T)$ policy is a random variable. Thus in general, the local $(s, T)$ policy is not a special case of the echelon $(s, T)$ policy. Nevertheless, if the reorder intervals and the lead times of a serial system satisfy $\sum_{i=2}^{j}\left(T_{i-1} \ominus L_{i}\right)=0$, then we can find an equivalent echelon policy by setting $S_{j}^{e}=S_{j}=$ $\sum_{i=1}^{j} s_{i}$, for all $j$.

With Proposition 1 , we can replace $\operatorname{IOP}_{j}(\cdot)$ in (3) with $S_{j}-D\left[t-\sum_{i=2}^{j}\left(T_{i-1} \ominus L_{i}\right), t\right)$ and use (3)-(5) to obtain the distribution for $I L_{j}$ and $I L_{j}^{-}$. For simplicity, we write $I P_{j}\left(\left\lfloor\tau / T_{j}\right\rfloor T_{j}\right)$ for $I P_{j}\left(t+L_{[j+1, N]}+\left\lfloor\tau / T_{j}\right\rfloor T_{j}\right)$, $I L_{j}^{-}(\tau)$ for $I L_{j}^{-}\left(t+L_{[j, N]}+\tau\right)$, and $I L_{j}(\tau)$ for $I L_{j}(t+$ $\left.L_{[j, N]}+\tau\right)$. The inventory dynamics in (3), (4), and (5) can be simplified as follows:

For $\tau=0, \ldots, T_{N}-1$,

$$
\begin{gathered}
I P_{j}\left(\left\lfloor\frac{\tau}{T_{j}}\right\rfloor T_{j}\right)=\min \left\{S_{j}-D\left[\sum_{i=2}^{j}\left(T_{i-1} \ominus L_{i}\right)\right),\right. \\
\left.I L_{j+1}^{-}\left(\left\lfloor\frac{\tau}{T_{j}}\right\rfloor T_{j}\right)\right\}, \\
I L_{j}^{-}(\tau)=I P_{j}\left(\left\lfloor\frac{\tau}{T_{j}}\right\rfloor T_{j}\right)-D\left[L_{j}+\mathbb{M}_{T_{j}}(\tau)\right), \\
I L_{j}(\tau)=I P_{j}\left(\left\lfloor\frac{\tau}{T_{j}}\right\rfloor T_{j}\right)-D\left[L_{j}+\mathbb{M}_{T_{j}}(\tau)\right] .
\end{gathered}
$$

Because the system starts each cycle when stage $N$ places an order, the system is a regenerative process with a cycle length of $T_{N}$ periods. Thus the longrun average inventory holding and backorder cost per period is equal to the expected inventory holding and backorder cost incurred in a cycle divided by the cycle length $T_{N}$. That is,

$G(\mathbf{s}, \mathbf{T})=\frac{1}{T_{N}} \mathrm{E}\left[\sum_{\tau=0}^{T_{N}-1}\left(\sum_{j=1}^{N} h_{j} I L_{j}(\tau)+\left(b+h_{[1, N]}\right)\left[I L_{1}(\tau)\right]^{-}\right)\right]$.

We next compute the average total fixed cost per period. Notice that stage $j$ will place an order at an order epoch $t$ when the total orders received between two consecutive order epochs in periods $t-T_{j}$ and $t$ is greater than zero.
Proposition 2. At each order epoch $t$, the order quantity for stage $j$ under the local $(s, T)$ policy is equal to $D\left[t-\sum_{i=2}^{j}\left(T_{i-1} \ominus L_{i}\right)-T_{j}, t-\sum_{i=2}^{j}\left(T_{i-1} \ominus L_{i}\right)\right)$.

At steady state, the probability that stage $j$ will place an order at an order epoch is $p_{j}=\mathrm{P}\left(D\left[T_{j}\right)>0\right)$. Thus the average order cost per period is $\sum_{j=1}^{N}\left(k_{j} p_{j} / T_{j}\right)$, which is the same as that for the echelon $(s, T)$ policy. This is intuitive: the order quantity for stage $j$ under an echelon $(s, T)$ policy is $D\left[t-T_{j}, t\right)$, which also covers $T_{j}$ periods of demand. The only difference is that the coverage is shifted by $\sum_{i=2}^{j}\left(T_{i-1} \ominus L_{i}\right)$ periods in the local policy. Consequently, both policies should have the same average fixed order cost per period in the long run when they have the same replenishment intervals.

With these results, the average total cost per period is

$$
\begin{aligned}
C(\mathbf{s}, \mathbf{T})=\sum_{j=1}^{N} \frac{k_{j} p_{j}}{T_{j}}+\frac{1}{T_{N}} \mathrm{E} & {\left[\sum _ { \tau = 0 } ^ { T _ { N } - 1 } \left(\sum_{j=1}^{N} h_{j} I L_{j}(\tau)\right.\right.} \\
& \left.\left.+\left(b+h_{[1, N]}\right)\left[I L_{1}(\tau)\right]^{-}\right)\right],
\end{aligned}
$$

where $\mathbf{s}=\left(s_{1}, \ldots, s_{N}\right)$ and $\mathbf{T}=\left(T_{1}, \ldots, T_{N}\right)$.

Below we provide a convenient recursion to evaluate $C(\mathbf{s}, \mathbf{T})$. The idea is similar to the bottom-up evaluation scheme for the echelon $(s, T)$ policy in (1) and (2). At each iteration, we evaluate the average inventory holding and backorder costs for echelon $j$, referred to as $G_{j}\left(y, \mathbf{T}_{\mathbf{j}}\right)$, provided that stage $j$ 's induced echelon base-stock level is equal to $y$ and its downstream stage $i(<j)$ follows a local $(s, T)$ policy with base-stock level $s_{i}$.

Proposition 3. Define

$$
\begin{aligned}
G_{1}\left(y, \mathbf{T}_{1}\right)=\frac{1}{T_{1}}( & \sum_{l=0}^{T_{1}-1} \mathrm{E}\left[h_{1}\left(y-D\left[L_{1}+l\right]\right)\right. \\
& \left.\left.+\left(b+h_{[1, N]}\right)\left(y-D\left[L_{1}+l\right]\right)^{-}\right]\right) .
\end{aligned}
$$

For $j=2, \ldots, N$,

$G_{j}\left(y, \mathbf{T}_{\mathbf{j}}\right)$

$=\frac{1}{T_{j}} \sum_{l=0}^{T_{j}-1} \mathrm{E}\left[h_{j}\left(y-D\left[\sum_{i=2}^{j} T_{i-1} \ominus L_{i}\right)-D\left[L_{j}+l\right]\right)\right.$ 


$$
\begin{array}{r}
+G_{j-1}\left(\operatorname { m i n } \left\{S_{j-1},\left(y-D\left[T_{j-1} \ominus L_{j}\right)\right.\right.\right. \\
\left.\left.\left.\left.-D\left[L_{j}+\left\lfloor\frac{l}{T_{j-1}}\right\rfloor T_{j-1}\right)\right)\right\}, \mathbf{T}_{\mathbf{j}-1}\right)\right],
\end{array}
$$

where $S_{j}=\sum_{i=1}^{j} s_{i}$. Then $\quad C(\mathbf{s}, \mathbf{T})=\sum_{j=1}^{N}\left(k_{j} p_{j} / T_{j}\right)+$ $G_{N}\left(S_{N}, \mathbf{T}_{\mathbf{N}}\right)$.

We now turn to optimization. We first show how to find the optimal local base-stock levels for fixed $\mathbf{T}=\left(T_{1}, \ldots, T_{N}\right)$. With fixed $\mathbf{T}$, the total average fixed $\operatorname{cost} \sum_{j=1}^{N}\left(k_{j} p_{j} / T_{j}\right)$ is a constant, so we only need to minimize $G_{N}\left(S_{N}, \mathbf{T}_{\mathrm{N}}\right)$.

We provide an observation below. Define $\tilde{G}_{1}^{e}\left(\cdot, T_{1}\right)=$ $G_{1}^{e}\left(\cdot, T_{1}\right)$. For $j=2, \ldots, N$, define

$$
\begin{aligned}
& \tilde{G}_{j}^{e}\left(y, \mathbf{T}_{\mathbf{j}}\right) \\
&=\frac{1}{T_{j}} \sum_{l=0}^{T_{j}-1} \mathrm{E}\left[h_{j}\left(y-D\left[\left(T_{j-1} \ominus L_{j}\right)+L_{j}+l\right]\right)\right. \\
&+\tilde{G}_{j-1}^{e}\left(\operatorname { m i n } \left\{S_{j-1},\left(y-D\left[\left(T_{j-1} \ominus L_{j}\right)\right.\right.\right.\right. \\
&\left.\left.\left.\left.\left.+L_{j}+\left\lfloor\frac{l}{T_{j-1}}\right\rfloor T_{j-1}\right)\right)\right\}, \mathbf{T}_{\mathbf{j}-\mathbf{1}}\right)\right] .
\end{aligned}
$$

Note that $\tilde{G}_{j}^{e}$ is the echelon cost function for a serial system with echelon base-stock levels $S_{j}^{e}=S_{j}=\sum_{i=1}^{j} S_{i}$ and with lead times $L_{1}$ for stage 1 and $L_{j}+\left(T_{j-1} \ominus L_{j}\right)$ for stage $j, j=2, \ldots, N$. Let $\tilde{C}^{e}(\mathbf{S}, \mathbf{T})$ be the average total cost per period for this modified system. It can be verified that

$$
\begin{gathered}
G_{j}\left(y, \mathbf{T}_{\mathbf{j}}\right)=\tilde{G}_{j}^{e}\left(y, \mathbf{T}_{\mathbf{j}}\right), \quad j=1,2, \\
G_{j}\left(y, \mathbf{T}_{\mathbf{j}}\right)=\tilde{G}_{j}^{e}\left(y, \mathbf{T}_{\mathbf{j}}\right)-\sum_{i=2}^{j-1} h_{[i+1, j]}\left(T_{i-1} \ominus L_{i}\right) \mu, \\
j=3, \ldots, N .
\end{gathered}
$$

This result implies the following proposition.

Proposition 4.

$$
C(\mathbf{s}, \mathbf{T})=\tilde{C}^{e}(\mathbf{S}, \mathbf{T})-\sum_{i=2}^{N-1} h_{[i+1, N]}\left(T_{i-1} \ominus L_{i}\right) \mu .
$$

Proposition 4 states that the average total cost of a system with a local $(s, T)$ policy is equal to that of a system with modified lead times and operated under the echelon $(s, T)$ policy with $S_{j}^{e}=\sum_{i=1}^{j} S_{i}$ minus a term that is independent of the local base-stock levels. Thus the optimization procedure of finding $S_{j}^{e}\left(\mathbf{T}_{\mathbf{j}}\right)$ in (1) and (2) can be applied to (10) and (11) to find the optimal induced echelon base-stock levels that minimize $G_{N}\left(S_{N}, \mathbf{T}_{\mathrm{N}}\right)$. Call the resulting optimal solution $\left(S_{1}\left(T_{1}\right), \ldots, S_{N}\left(\mathbf{T}_{\mathbf{N}}\right)\right)$. The optimal local base-stock levels can be found by setting $s_{1}^{*}\left(T_{1}\right)=S_{1}\left(T_{1}\right)$, and $s_{j}^{*}\left(\mathbf{T}_{\mathbf{j}}\right)=S_{j}\left(\mathbf{T}_{\mathbf{j}}\right)-S_{j-1}\left(\mathbf{T}_{\mathbf{j}-1}\right), j=$ $2, \ldots, N$. Let $\mathbf{s}^{*}(\mathbf{T})=\left(s_{1}^{*}\left(T_{1}\right), \ldots, s_{j}^{*}\left(\mathbf{T}_{\mathbf{j}}\right)\right)$.

Based on the preceding observation, the following result is immediate.

Proposition 5. For fixed $\mathbf{T}, \mathbf{s}^{*}(\mathbf{T})$ is the optimal local base-stock vector.

We next show how to optimize the reorder intervals for the local $(s, T)$ policy. We need one additional result for this purpose. Recall the conclusion obtained from Proposition 1: The local $(s, T)$ policy is not a special case of the echelon $(s, T)$ policy. A natural question is, which policy has a smaller optimal cost? From our discussion, it is conceivable that the local policy is less effective because of the delay of demand information. We shall confirm this conjecture below.

Proposition 6.

(1) $S_{j}^{e}\left(\mathbf{T}_{\mathbf{j}}\right) \leq S_{j}\left(\mathbf{T}_{\mathbf{j}}\right)=\sum_{i=1}^{j} S_{i}^{*}$ for $j=1, \ldots, N$.

(2) For fixed $\mathbf{T}, G_{N}\left(S_{N}\left(\mathbf{T}_{\mathbf{N}}\right), \mathbf{T}_{\mathbf{N}}\right) \geq G_{N}^{e}\left(S^{e}\left(\mathbf{T}_{\mathbf{N}}\right), \mathbf{T}_{\mathbf{N}}\right)$.

(3) For fixed $\mathbf{T}, C\left(\mathbf{s}^{*}(\mathbf{T}), \mathbf{T}\right) \geq C^{e}\left(\mathbf{S}^{\mathbf{e}}(\mathbf{T}), \mathbf{T}\right)$.

Proposition 6(1) states that the optimal induced echelon base-stock level for the local policy is no less than that for the echelon policy. This is intuitive because the local policy requires more stocks to cover the unobserved demand due to the information delay. Parts (2) and (3) of Proposition 6 state that for a given $\mathbf{T}$, the optimal cost of the local policy, excluding and including the fixed ordering cost, respectively, is higher than or equal to that of the echelon policy. Suppose that the optimal reorder intervals for the local policy are $\mathbf{T}^{*}$. From Proposition 6(3), the optimal cost of the local policy must be greater than or equal to the optimal cost of the echelon policy that uses the same $\mathbf{T}^{*}$. The latter is clearly greater than or equal to the optimal cost of the echelon policy. Therefore, we have the following result:

Proposition 7. The best echelon $(s, T)$ policy always dominates the best local $(s, T)$ policy. 
Proposition 6 leads to an approach to find $\mathbf{T}^{*}$. Shang and Zhou (2009) proposed an approach to find bounds for the optimal echelon reorder intervals. Then a complete enumeration is conducted to search for the optimal reorder intervals. Our approach is based on the same idea. More specifically, let $C^{h}$ be the cost of a heuristic local policy, and define $C\left(\mathbf{T}^{*}\right)=C\left(\mathbf{s}^{*}\left(\mathbf{T}^{*}\right), \mathbf{T}^{*}\right)$, $C^{e}\left(\mathbf{T}^{*}\right)=C^{e}\left(\mathbf{S}^{\mathbf{e}}\left(\mathbf{T}^{*}\right), \mathbf{T}^{*}\right), G_{N}^{e}\left(\mathbf{T}_{\mathbf{N}}^{*}\right)=G_{N}^{e}\left(S_{N}^{e}\left(\mathbf{T}_{\mathbf{N}}^{*}\right), \mathbf{T}_{\mathbf{N}}^{*}\right)$. We can find bounds for $T_{j}^{*}$ from the following inequalities. For $j=1,2, \ldots, N$,

$$
\begin{aligned}
C^{h} & \geq C\left(\mathbf{T}^{*}\right) \geq C^{e}\left(\mathbf{T}^{*}\right)>G_{N}^{e}\left(\mathbf{T}_{\mathbf{N}}^{*}\right) \\
& \geq \sum_{i=j+1}^{N} \underline{c}_{i}\left(T_{i}^{*}\right)+G_{j}^{l}\left(T_{j}^{*}\right)+\pi_{j} .
\end{aligned}
$$

The second inequality in (12) follows from Proposition 6(2). The $\underline{c}_{i}(\cdot)$ and $G_{j}^{l}(\cdot)$ functions and the last inequality in (12) were established in Shang and Zhou (2009). We provide a brief explanation below: $\underline{c}_{j}\left(T_{j}\right)$ is a lower bound for stage $j^{\prime}$ s cost. It is a function of $T_{j}$ and is independent of $T_{i}, i \neq j . \pi_{j}=$ $\sum_{i=2}^{j}\left(h_{i} \mathrm{E}\left[D\left[L_{[1, i-1]}\right)\right]\right)$, the average inventory in-transit cost. $G_{j}^{l}\left(T_{j}\right)=\min _{y} G_{j}^{l}\left(y, T_{j}\right)$, where

$$
\begin{aligned}
G_{j}^{l}\left(y, T_{j}\right)=\frac{1}{T_{j}} \sum_{t=0}^{T_{j}} \mathrm{E} & h_{j}\left(y-D\left[L_{[1, j]}+t\right]\right) \\
& \left.+\left(b+h_{[j, N]}\right)\left(y-D\left[L_{[1, j]}+t\right]\right)^{-}\right] .
\end{aligned}
$$

Shang and Zhou (2009) showed that $G_{j}^{l}\left(T_{j}\right)$ is convex in $T_{j}$.

The procedure starts from stage $N$. For $j=N$, Equation (12) implies

$$
G_{N}^{l}\left(T_{N}^{*}\right)<C^{h}-\pi_{N} .
$$

From this inequality, one can obtain an upper bound, $\bar{T}_{N}$, for $T_{N}^{*}$. Clearly, a lower bound is equal to one.

Next, for $j=N-1$, Equation (12) becomes

$$
\underline{c}_{N}\left(T_{N}^{*}\right)+G_{N-1}^{l}\left(T_{N-1}^{*}\right)+\pi_{N-1}<C^{h} .
$$

To search for bounds for $T_{N-1}^{*}$ by using $G_{N-1}^{l}(\cdot)$, we need to find the minimum value for $\underline{c}_{N}\left(T_{N}\right)$. Unfortunately, $\underline{c}_{j}\left(T_{j}\right)$ is not convex, so one needs to search over $\left[1, \bar{T}_{N}\right]$ to find the minimum cost of $\underline{c}_{N}\left(T_{N}\right)$, referred to as $\underline{\underline{c}}_{N}$. Consequently, (13) can be rewritten as

$$
G_{N-1}^{l}\left(T_{N-1}^{*}\right)<C^{h}-\underline{c}_{N}-\pi_{N-1} .
$$

From this, one can obtain an upper bound $\bar{T}_{N-1}$ for $T_{N-1}^{*}$. Again, we set the lower bound equal to one, and for $T_{N-1} \in\left[1, \bar{T}_{N-1}\right]$, we can search for the minimum $\underline{c}_{N-1}\left(T_{N-1}\right)$, refereed to as $\underline{c}_{N-1}$. The procedure repeats until $j=1$. At the end of the procedure, we find the solution bounds for $T_{j}^{*}, j=1, \ldots, N$. To find the optimal local replenishment intervals, we only need to search all the feasible solutions $1 \leq T_{j} \leq \bar{T}_{j}, j=1, \ldots, N$, that satisfy integer-ratio constraints.

\section{Numerical Study}

The goal of the numerical study is to examine the cost improvement by implementing each of the suggested strategies. This is accomplished by comparing the cost improvement between two pairs of the inventory policies: local $(s, T)$ policy versus echelon $(s, T)$ policy and local $(s, T)$ policy versus local $(r, n Q)$ policy.

To strengthen the creditability of our numerical conclusions, we design cost parameters based on Willems (2008), where he collected cost data from 38 supply chains. He classified supply chain stages into five categories: Dist, Manuf, Part, Retail, and Trans. (We refer the reader to Willems 2008 for the definitions.) One observation is useful: the top three types of stages that have the largest direct cost are the Part, Manuf, and Dist stages. These stages account for about $95 \%$ of the total supply chain direct cost on average. More specifically, the Part stage accounts for about $60 \%$; the Manuf stage, $25 \%$; and the Dist stage, $10 \%$. Because inventory holding cost is related to the direct cost, we shall use this observation as a guideline to design the holding cost parameters.

We consider a three-stage system that represents these top three stages. Without loss of generality, we fix the total supply chain holding $\operatorname{cost} h_{[1,3]}$ at one and assume the following holding cost parameters:

$$
\begin{array}{r}
\left(h_{1}, h_{2}, h_{3}\right) \in\{(0.05,0.15,0.8),(0.05,0.8,0.15), \\
(0.15,0.35,0.5),(0.15,0.5,0.35)\} .
\end{array}
$$

The first choice, for example, can be viewed as a supply chain with Part at stage 3, Manuf at stage 2, and Dist at stage 1. In general, these holding cost patterns are consistent with Willems's (2008) finding that an upstream stage has a higher direct cost, which implies a relatively higher holding cost. 
With the same spirit that most cost is incurred at one stage, we fix the total system fixed order cost to 10 and 100 and allocate these total costs into each stage according to $(20 \%, 20 \%, 60 \%)$ ratios. Thus the resulting fixed cost parameters are

$$
\begin{gathered}
\left(k_{1}, k_{2}, k_{3}\right) \in\{(2,2,6),(2,6,2),(6,2,2),(20,20,60), \\
(20,60,20),(60,20,20)\} .
\end{gathered}
$$

We consider a service level approximately equal to $90 \%$ and $97.5 \%$, which corresponds to

$$
b \in\{9,39\} .
$$

We assume that the demand follows a stuttering Poisson demand process (e.g., Hadley and Whitin 1963, Chen and Zheng 1998), one type of compound Poisson demand in which the arrival process is Poisson with rate $\lambda$ and the demand size follows a geometric distribution, i.e.,

$$
\mathrm{P}(U=x)=(1-\alpha)^{x-1} \alpha, \quad x=1,2, \ldots,
$$

where $U$ is the demand size of a customer and $0<$ $\alpha \leq 1$. (When $\alpha=1$, the demand reduces to Poisson.) The mean and squared coefficient of variation (scv) of the total demand in one period is $\lambda / \alpha$ and $(2-\alpha) / \lambda$, respectively. We set $\lambda / \alpha=5$ with $\mathrm{scv}=1 / 3$ (i.e., $\alpha=$ $3 / 4$ and $\lambda=15 / 4)$, representing smaller demand variability, and scv $=1$ (i.e., $\alpha=1 / 3, \lambda=5 / 3$ ), representing higher demand variability.

Finally we consider two lead time settings, short and long:

$$
\left(L_{1}, L_{2}, L_{3}\right) \in\{(1,1,1),(3,3,3)\} .
$$

The preceding system parameters generate a total number of 192 instances. For each of the instances, we compute the exact optimal solution for each of the policies. For simplicity, let $C_{L Q}, C_{E T}$, and $C_{L T}$ represent the optimal costs for the local $(r, n Q)$, echelon $(s, T)$, and local $(s, T)$ policies, respectively. ${ }^{3}$ We define the percentage cost reduction as below:

$$
\Delta_{i} \%=\frac{C_{L T}-C_{i}}{C_{L T}} \times 100 \%, \quad i \in\{E T, L Q\} .
$$

Table 1 summarizes the average, maximum, and minimum percentage cost reduction in these 192 instances.

\footnotetext{
${ }^{3}$ We use the optimal echelon batch sizes (reorder intervals) and their corresponding optimal local reorder points (base-stock levels) as a heuristic solution to obtain $C^{h}$ when we search for the optimal local $(r, n Q)((s, T))$ policy.
}

Table 1 Percentage Cost Reduction by Adopting Two Strategies

\begin{tabular}{lccc}
\hline & Average & Maximum & Minimum \\
Cost reduction & $\Delta(\%)$ & $\Delta(\%)$ & $\Delta(\%)$ \\
\hline Local $(s, T)$ vs. local $(r, n Q)$ policy & 11.27 & 28.32 & 1.75 \\
Local $(s, T)$ vs. echelon $(s, T)$ policy & 5.51 & 16.67 & 0.00 \\
\hline
\end{tabular}

From the table, the average (maximum) cost reduction when the system switches from the local $(s, T)$ policy to the local $(r, n Q)$ policy is $\Delta_{L Q}=11.27 \%$ $(28.32 \%)$, which is significantly larger than the reduction of $\Delta_{E T}=5.51 \%(16.67 \%)$ when switching to the echelon $(s, T)$ policy. This result suggests that implementing a more flexible delivery policy is more beneficial than acquiring the real-time demand information. It is interesting to further examine this conclusion under different demand variabilities. Figure 3 presents $\Delta_{L Q}$ and $\Delta_{E T}$ for a three-stage system with $\left(h_{1}, h_{2}, h_{3}\right)=$ $(0.05,0.15,0.8),\left(k_{1}, k_{2}, k_{3}\right)=(60,20,20), b=9$, and $L_{1}=$ $L_{2}=L_{3}=1$ under $\mathrm{scv}=1 / 3,2 / 3,1,4 / 3$, and $5 / 3$. It can be seen that the system indeed benefits more by adopting an agile logistics when demand is more variable. This observation holds true for the other sets of parameters.

The above observation provides an important insight: when demand becomes more variable, managers usually want to invest in IT systems to acquire real-time demand information. However, acquiring demand information alone may not be an effective strategy; it is necessary to implement an agile logistics system to achieve supply chain efficiency.

Our model and the inventory policies have been used to quantify the benefits of demand information and flexible delivery. Sections 4.1 and 4.2 provide a more detailed discussion on these benefits. Section 4.3

Figure 3 The Impact of Demand Variability on $\Delta_{L Q}$ and $\Delta_{E T}$

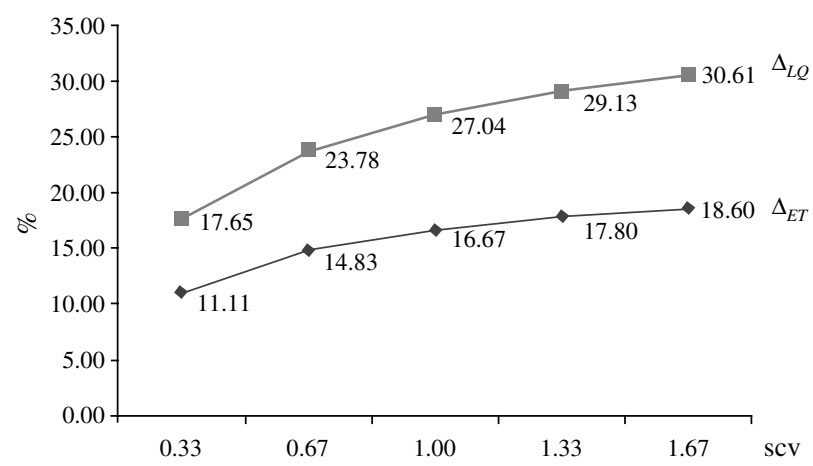


discusses the effectiveness of inferring reorder intervals from the optimal batch sizes.

\subsection{Value of Demand Information}

We numerically illustrated that it is more beneficial for a system to move from the local $(s, T)$ policy to the local $(r, n Q)$ policy than to the echelon $(s, T)$ policy. It is natural to ask how much additional benefit can be obtained by adding demand information to the local $(r, n Q)$ policy. Thus to assess this additional benefit, we need to compare the optimal cost between the local and echelon $(r, n Q)$ policies.

We define the VOI under the $(r, n Q)$ policy as

$$
V O I_{Q}=\frac{C_{L Q}-C_{E Q}}{C_{L Q}} \times 100 \%
$$

where $C_{E Q}$ represents the optimal cost of an echelon $(r, n Q)$ policy. We refer the reader to Chen and Zheng (1998) or Shang and Zhou (2009) for an approach to find the optimal solution.

In our test bed of 192 instances, the average (maximum) $V O I_{Q}$ is $0.55 \%(2.71 \%)$. This result suggests that the additional benefit from the demand information is surprisingly minimal in a system with flexible deliveries. Note that this conclusion is made by comparing the exact optimal local and echelon $(r, n Q)$ policies. Numerically, we may obtain a very large VOI if both local and echelon policies are not optimized. For example, Chen (1998b) arbitrarily chose the same batch sizes for both local and echelon $(r, Q)$ policies. In his numerical study for three-stage systems, the average (maximum) $\mathrm{VOI}_{Q}$ is $1.61 \%(7.5 \%)$. Based on our numerical experience, the $V O I_{Q}$ tends to be small if we fix both local and echelon batch sizes to the optimal batch sizes obtained from the echelon $(r, n Q)$ policy. In our test bed, the average (maximum) VOI then is $0.65 \%(3.99 \%)$. Clearly, the difference between $0.65 \%$ and $0.55 \%$ is due to the effect of optimizing local batch sizes.

Notice that the average $\Delta_{E T}=5.51 \%$ obtained earlier is the cost reduction by switching from the local $(s, T)$ policy to the echelon $(s, T)$ policy. This is equivalent to the VOI under the less flexible $(s, T)$ policy. Thus one insight learned from this study is that how much VOI a supply chain can achieve depends on how flexible its logistics system is.
Figure 4 Sensitivity Analysis: Average Value of Demand Information
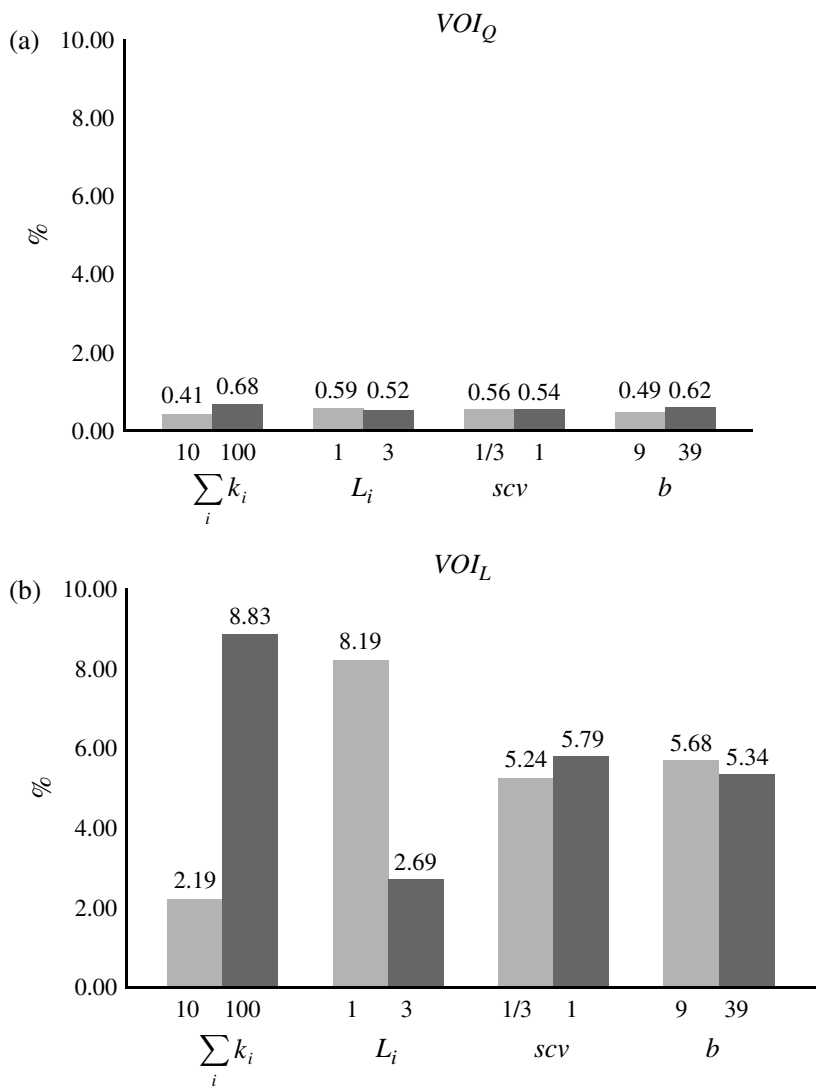

We end this section by identifying the conditions under which the system benefits most when acquiring the demand information under each of the two logistics settings. For a comparable presentation, denote $V O I_{T}=\Delta_{E T}$. We illustrate some comparative statics of $V O I_{Q}$ and $V O I_{T}$ in Figure 4.

For the $(r, n Q)$ policy, the average $V O I_{Q}$ increases from $0.41 \%$ to $0.68 \%$ when the total fixed costs $\sum_{i} k_{i}$ change from 10 to 100 . Also, the average $V O I_{Q}$ tends to increase as the backorder cost $b$ increases. It can be seen from Figure 4(a) that the system lead times and demand variability have a relatively smaller impact on the $V O I_{Q}$.

For the $(s, T)$ policy, the $V O I_{T}$ mainly depends on how much demand information is delayed, i.e., $D\left[\sum_{i=2}^{j}\left(T_{i-1} \ominus L_{i}\right)\right]$ for each stage $j$. When the lead times $L_{i}$ are short and reorder intervals $T_{i}, i<N$ are long, such that $T_{i-1}>L_{i}$, the delayed demand information $D\left[\sum_{i=2}^{j}\left(T_{i-1} \ominus L_{i}\right)\right]$ becomes larger. Thus it is more beneficial to provide the demand information under 
such conditions. To see this, we compare one instance with short lead times $\left(L_{1}, L_{2}, L_{3}\right)=(1,1,1)$ to another instance with long lead times $\left(L_{1}, L_{2}, L_{3}\right)=(3,3,3)$; the other parameters are the same:

$$
\begin{gathered}
\left(k_{1}, k_{2}, k_{3}\right)=(60,20,20), \\
\left(h_{1}, h_{2}, h_{3}\right)=(0.15,0.5,0.35), \\
b=39, \quad \alpha=3 / 4 .
\end{gathered}
$$

For both cases, the optimal reorder intervals for the echelon and local policies are $(7,7,7)$ and $(6,6,6)$, respectively. The corresponding $V O I_{T}$ is $11.07 \%$ for the short lead time case and $6.87 \%$ for the long lead time case. Finally, the $V O I_{T}$ increases when $\sum_{i} k_{i}$ is larger. In our test, when $\sum_{i} k_{i}=10$, the average $V O I_{T}$ is $2.19 \%$, and when $\sum_{i} k_{i}=100$, the average $V O I_{T}$ increases to $8.83 \%$. This is because higher fixed costs increase the length of the resulting reorder intervals, which in turn delays the demand information more significantly.

\subsection{Value of Flexible Delivery}

Recall the average $\Delta_{L Q}$ is $11.27 \%$ in our test bed. This is the value of flexible delivery (VOD for short) when the system only has local information. For this reason, let us denote $V O D_{L}=\Delta_{L Q}$. Similarly, we can obtain the average VOD when the system has full information, i.e., the cost reduction of switching from the echelon $(s, T)$ policy to the echelon $(r, n Q)$ policy. Denote the VOD under echelon information as

$$
V O D_{E}=\frac{C_{E T}-C_{E Q}}{C_{E T}} \times 100 \% .
$$

It is conceivable that the average $V O D_{E}$ should be lower than the average $V O D_{L}$ because a more responsive delivery speeds up not only the material flow but also the demand information flow. Our numerical study confirms this intuition: In our test bed, the average $V O D_{E}=6.76 \%$ and $V O D_{L}=11.27 \%$. In general, flexible delivery reduces total cost significantly, regardless of the information setting.

We provide some comparative statics of $V O D_{E}$ and $V O D_{L}$ in Figure 5 and examine the conditions under which the system benefits more by switching from the $(s, T)$ policy to the $(r, n Q)$ policy. For the echelon information, we observe that when the lead times $L_{i}$ are shorter (longer), the fixed costs $k_{i}$ are larger
Figure 5 Sensitivity Analysis: Average Value of Flexible Delivery
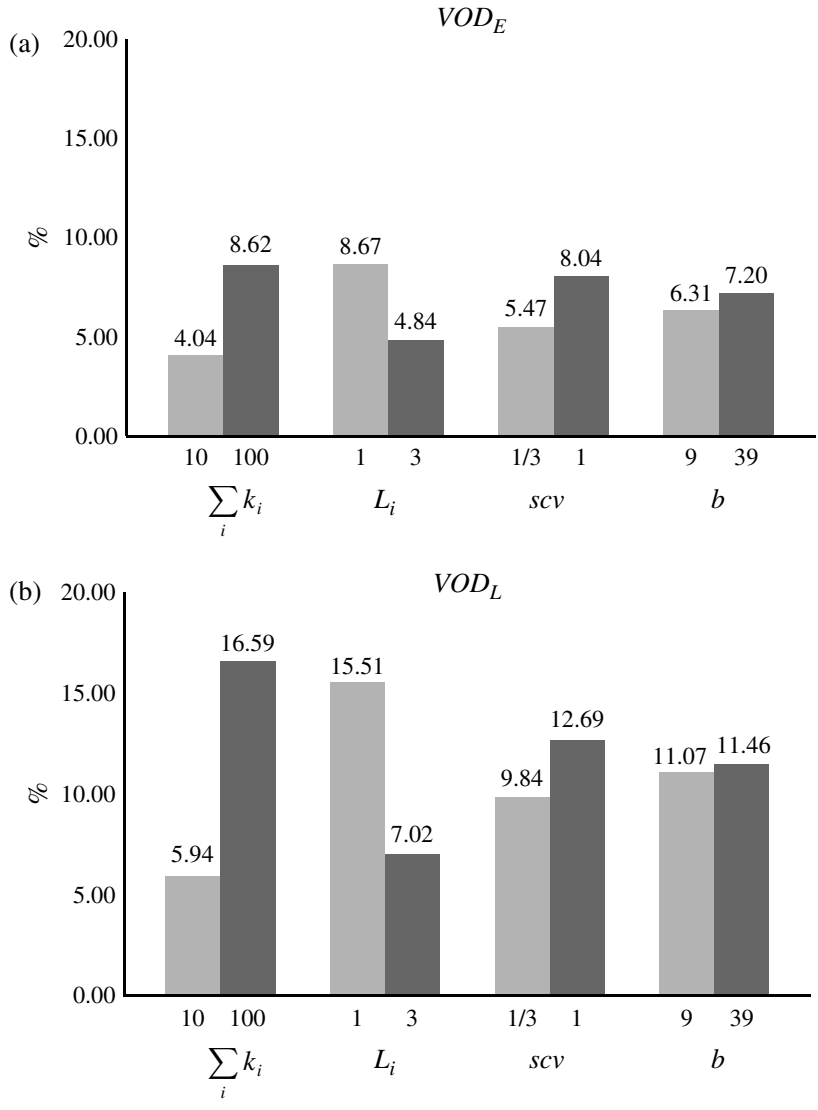

(smaller), the backorder cost $b$ is larger (smaller), or the demand is more (less) variable, the $V O D_{E}$ tends to be higher (lower). (See Figure 5 for the exact cost reduction percentage.)

We explain the above observation below. Because both echelon policies have the same demand information, the cost benefit of the $(r, n Q)$ policy depends on how flexible it can retain. Larger fixed costs $k_{i}$ will lead to longer replenishment intervals $T_{i}$ and therefore the $(s, T)$ policy becomes less flexible. This increases the benefit of switching to the $(r, n Q)$ policy. If $L_{i}$ is shorter, the flexibility of $(r, n Q)$ policy becomes more prominent, and therefore the corresponding $V O D_{E}$ is larger. The last two observations are intuitive. When the backorder penalty or demand variance increases, the system benefits more from the flexible $(r, n Q)$ policy in that it can better respond to the demand to avoid higher costs. Thus the average $V O D_{E}$ is higher when $b$ or scv is larger. 
In the local information scenario, similar to the echelon information, we observe that when the fixed costs $k_{i}$ are high, the lead times $L_{i}$ are short, the backorder cost $b$ is high, or demand is more variable, the $V O D_{L}$ tends to be high. The intuition behind these observations is similar to what we discussed for the echelon information scenario. However, the impact of the fixed costs and lead times is considerably larger on $V O D_{L}$ than on $V O D_{E}$. This observation suggests that if the fixed costs of the system are large or the lead times are short, the system can improve significantly by switching to a more flexible delivery policy even without real-time demand information.

Figure 6 summarizes the cost reduction achieved by each of the inventory policies by switching from the local $(s, T)$ policy. The symbol " $\rightarrow$ " represents switching from one policy to another. The number next to the arrow represents the average percentage cost reduction. For example, $11.75 \%$ is the cost reduction achieved by switching system from the local $(s, T)$ policy to the echelon $(r, n Q)$ policy. This $11.75 \%$ is the maximum cost reduction that the supply chain system can achieve. Implementing a more responsive delivery policy (e.g., the local $(r, n Q)$ policy) recovers about $95.91 \%(=11.27 / 11.75 \times 100 \%)$ of the maximum cost reduction. On the other hand, acquiring realtime demand information only recovers about $46.89 \%$ $(=5.51 / 11.75 \times 100 \%)$.

\subsection{Effectiveness of Inferring Reorder Intervals from Batch Sizes}

In the deterministic demand model, the reorder interval $T_{j}$ and the batch size $Q_{j}$ are equivalent. That is, the reorder interval can be inferred by the batch size with the following equation: $T_{j}=Q_{j} / \mu$, where $\mu$ is the demand rate. In this section, we aim to examine

Figure 6 Cost Comparisons of the Considered Four Inventory Policies

\begin{tabular}{|c|c|c|c|c|c|}
\hline & \multicolumn{2}{|c|}{$\begin{array}{c}\text { With real-time } \\
\text { demand information }\end{array}$} & & \multicolumn{2}{|c|}{$\begin{array}{c}\text { Without real-time } \\
\text { demand information }\end{array}$} \\
\hline \multirow{2}{*}{$\begin{array}{l}\text { Flexible } \\
\text { delivery policy }\end{array}$} & \multicolumn{2}{|c|}{ Echelon $(r, n Q)$ policy } & $\mathrm{VOI}_{Q}$ & \multicolumn{2}{|c|}{ Local $(r, n Q)$ policy } \\
\hline & $V O D_{E}$ & $6.76 \%$ & & $V O D_{L}$ & $11.27 \%$ \\
\hline \multirow{2}{*}{$\begin{array}{l}\text { Periodic } \\
\text { delivery policy }\end{array}$} & \multirow{2}{*}{\multicolumn{2}{|c|}{ Echelon $(s, T)$ policy }} & $\mathrm{VOI}_{T}$ & \multirow{2}{*}{\multicolumn{2}{|c|}{ Local $(s, T)$ policy }} \\
\hline & & & $5.51 \%$ & & \\
\hline
\end{tabular}

whether effective reorder intervals can be inferred by the optimal batch sizes $Q_{j}^{*}$ and the demand rate $\mu$.

We first explain how to obtain the inferred reorder intervals. Note that $Q_{j}^{*} / \mu$ may not be an integer. If this is the case, we round $Q_{j}^{*} / \mu$ into an integer, denoted as $\left\langle Q_{j}^{*} / \mu\right\rangle$, where $\langle\cdot\rangle$ is the standard rounding operator. If the resulting $\left\langle Q_{j}^{*} / \mu\right\rangle$ satisfies the integer-ratio constraints, then $\left\langle Q_{j}^{*} / \mu\right\rangle$ is the inferred reorder interval for stage $j$. Otherwise, starting from stage $j=1,2, \ldots, N$, we set $\left\langle Q_{j+1}^{*} / \mu\right\rangle$ equal to the closest integer multiple of $\left\langle Q_{j}^{*} / \mu\right\rangle$. For example, suppose that $\left(Q_{1}^{*}, Q_{2}^{*}, Q_{3}^{*}\right)=(27,27,54)$ and $\mu=5$. Then $\left(\left\langle Q_{1}^{*} / \mu\right\rangle,\left\langle Q_{2}^{*} / \mu\right\rangle,\left\langle Q_{3}^{*} / \mu\right\rangle\right)=(5,5,11)$, and the inferred reorder intervals are $(5,5,10)$.

Let $C_{E T}^{\prime}\left(C_{L T}^{\prime}\right)$ denote the total cost obtained by using the inferred echelon (local) reorder intervals and the resulting optimal echelon (local) base-stock levels. Let

$$
\epsilon_{i}=\frac{\left(C_{i}^{\prime}-C_{i}\right)}{C_{i}} \times 100 \%, \quad i \in\{E T, L T\}
$$

denote the percentage cost increase by using the inferred reorder intervals. We observe the following:

(1) There are 23 (36) out of 192 instances that the inferred echelon (local) reorder intervals are equal to the optimal echelon (local) reorder intervals. Thus from the solution perspective, it is in general not effective to infer the reorder intervals from the optimal batch sizes.

(2) Although the inferred local reorder intervals generate more optimal solutions than the inferred echelon ones, the average $\epsilon_{E T}$ of $1.16 \%$ is lower than the average $\epsilon_{L T}$ of $4.76 \%$. Hence the inferred reorder intervals are less effective when the system only has local information. In some cases, the $\epsilon_{L T}$ can be very large. For example, for the 12 instances with $b=39$, $\sigma=1 / 3, L_{j}=1$, and $\sum_{i=1}^{3} k_{i}=10$, the average $\epsilon_{L T}$ is $16.75 \%$.

(3) For both information settings, the inferred reorder intervals tend to be longer than the optimal ones. This implies that the optimal batch sizes are large in the $(r, n Q)$ system. This may be explained as follows: Large batch sizes have two opposite effects on the system performance. On the one hand, when batch sizes are large, the system can gain the benefit of economies of scale. On the other hand, large batch sizes will make the system order less frequently, which reduces the responsiveness to the demand. 
However, the latter effect is less significant in the $(r, n Q)$ policy because a stage can place an order in any period. Thus the former effect dominates and leads to larger optimal batch sizes in the $(r, n Q)$ model.

\section{Concluding Remarks}

This paper investigates how to improve the cost performance of a supply chain, in which each location can only access local information and replenish in fixed intervals. We model this supply chain by a serial inventory system with local $(s, T)$ policies. We consider two improvement strategies: implementing a flexible delivery policy (local $(r, n Q)$ policy) and acquiring real-time demand information (echelon $(s, T)$ policy). The inventory policies resulting from the improvement strategies are well studied in the literature. To compare costs in these scenarios, we analyze the local $(s, T)$ policy. The analysis includes policy evaluation and optimization. We show that the local $(s, T)$ policy is not a subset of the echelon $(s, T)$ policy and that the former incurs a higher system cost. Our numerical study suggests that implementing a more flexible delivery policy is more effective than acquiring the real-time demand information. We also identify the conditions under which the system is less beneficial for each of the improvement strategies. Certainly our conclusions are drawn without considering the potential benefits of implementing $(s, T)$ policies, such as ease of personnel scheduling and coordination, and without considering actual implementation and maintenance costs when implementing these strategies. Managers should therefore consider the insights obtained from our study as well as those unmodeled factors when designing the logistics and information systems of a supply chain. Finally, we find that inferring reorder intervals from optimal batch sizes may lead to significant cost inefficiency, especially when a supply chain only has local information.

\section{Acknowledgments}

The authors thank Gerard Cachon and the anonymous reviewers for helpful comments.

\section{Appendix A. Proofs}

For ease of exposition, throughout the appendix, we relax the integer assumption on inventory-related variables. As a result, all the functions arecontinuous and satisfy the condition of Leibniz's rule so that we can exchange the signs of derivative and expectation.

Proposition 1. We prove the proposition by induction. For stage 1 , by definition $s_{1}=S_{1}$, the result is clearly true. Suppose the result is true for $j=i-1$. Let $t$ be an order epoch for stage $i$. Then stage $i-1$ 's last order epoch before $t$ is $t-\left(T_{i-1} \ominus L_{i}\right)$, at which, by the inductive assumption, the echelon inventory position at stage $i-1$ is $S_{i-1}-D\left[t-\left(T_{i-1} \ominus L_{i}\right)-\sum_{j=2}^{i-1}\left(T_{j-1} \ominus L_{j}\right), t-\left(T_{i-1} \ominus L_{i}\right)\right)$. Furthermore, because stage $i-1$ will not place any order between time $t-\left(T_{i-1} \ominus L_{i}\right)$ and $t$, the echelon inventory order position at time $t$ for stage $j=i$ is

$$
\begin{aligned}
S_{i-1} & -D\left[t-\left(T_{i-1} \ominus L_{i}\right)-\sum_{j=2}^{i-1}\left(T_{j-1} \ominus L_{j}\right), t-\left(T_{i-1} \ominus L_{i}\right)\right) \\
& +s_{i}-D\left[t-T_{i-1} \ominus L_{i}, t\right)=S_{i}-D\left[t-\sum_{j=2}^{i}\left(T_{j-1} \ominus L_{j}\right), t\right),
\end{aligned}
$$

where the equality follows from the definition of $S_{i}$. This completes the induction proof.

Proposition 2. The lemma is clearly true for $j=1$. Suppose that it is true for stage $j-1$. For any time point $t$ that is an order epoch of stage $j$, the last order epoch before $t$ of stage $j$ is $t-T_{j}$. Note that the order quantity of stage $j$ at time $t$ is the total orders from stage $j-1$ in $\left[t-T_{j}, t\right]$. Because the orders between stages are synchronized, $t-T_{j}+L_{j}$ is an order epoch of stage $j-1$. Moreover, we know in $\left[t-T_{j}, t\right]$, stage $j-1$ will place $T_{j} / T_{j-1}$ orders. We only need to specify the time epoch of the first order from stage $j-1$ in this interval, which is $t-T_{j}-\left(T_{j-1} \ominus L_{j}\right)+T_{j-1}$. As a result, the last order from stage $j-1$ in this interval is at time $t-\left(T_{j-1} \ominus L_{j}\right)$. Therefore, by the inductive assumption, the demand covered in these orders is the demand in the interval $\left[t-T_{j}-\right.$ $\left.\sum_{i=2}^{j}\left(T_{i-1} \ominus L_{i}\right), t-\sum_{i=2}^{j}\left(T_{i-1} \ominus L_{i}\right)\right)$.

Proposition 3. The derivation of the formula for $C(\mathbf{s}, \mathbf{T})$ is straightforward. Below, we give a proof by induction for the recursion of $G_{j}\left(y, \mathbf{T}_{\mathbf{j}}\right)$. For notational convenience, we omit $\mathbf{T}_{\mathbf{j}}$ in $G_{j}\left(y, \mathbf{T}_{\mathbf{j}}\right)$ in this proof.

The derivation of the formula for $G_{1}(y)$ is straightforward. Now let $j \in\{2, \ldots, N\}$ and suppose that the formula for $G_{i}(y)$ has been proved for $i=1, \ldots, j-1$. We derive the formula for $G_{j}(y)$.

Suppose that $t$ is an order epoch of stage $j$. Then

$$
I P_{j}(t)=I O P_{j}(t)=y-D\left[t-\sum_{i=2}^{j}\left(T_{i-1} \ominus L_{i}\right), t\right],
$$

where the first equality follows from the assumption that stage $j+1$ has always sufficient inventory and the last 
equality follows from Proposition 2. This leads to the following echelon $j$ costs in the periods $t+L_{j}, \ldots, t+L_{j}+T_{j}-1$ :

$$
\begin{aligned}
\sum_{l=0}^{T_{j}-1} \mathrm{E} & {\left[h_{j}\left(I P_{j}(t)-D\left[t, t+L_{j}+l\right]\right)\right] } \\
= & \sum_{l=0}^{T_{j}-1} \mathrm{E}\left[h_{j}\left(y-D\left[t-\sum_{i=2}^{j}\left(T_{i-1} \ominus L_{i}\right), t\right]-D\left[t, t+L_{j}+l\right]\right)\right] \\
= & \sum_{l=0}^{T_{j}-1} \mathrm{E}\left[h_{j}\left(y-D\left[t-\sum_{i=2}^{j}\left(T_{i-1} \ominus L_{i}\right), t+L_{j}+l\right]\right)\right] .
\end{aligned}
$$

Next we look at the lower echelon costs, which may be obtained via $G_{j-1}(y)$ and $I P_{j-1}\left(t^{\prime}\right)$ in the periods $t^{\prime}=$ $t+L_{j}, t+L_{j}+T_{j-1}, \ldots, t+L_{j}+\left(T_{j} / T_{j-1}-1\right) T_{j-1}$. It holds that

$$
\begin{aligned}
& I P_{j-1}\left(t+L_{j}\right) \\
& =\min \left\{I O P_{j-1}\left(t+L_{j}\right), I L_{j}^{-}\left(t+L_{j}\right]\right\} \\
& =\min \left\{S_{j-1}-D\left[t+L_{j}-\sum_{i=2}^{j-1}\left(T_{i-1} \ominus L_{i}\right), t+L_{j}\right),\right. \\
& \left.I P_{j}(t)-D\left[t, t+L_{j}\right)\right\} \\
& =\min \left\{S_{j-1}-D\left[t+L_{j}-\sum_{i=2}^{j-1}\left(T_{i-1} \ominus L_{i}\right), t+L_{j}\right),\right. \\
& =\min \left\{S_{j-1}, y-D\left[t-\sum_{i=2}^{j}\left(T_{i-1} \ominus L_{i}\right), t+L_{j}-\sum_{i=2}^{j-1}\left(T_{i-1} \ominus L_{i}\right)\right)\right\} \\
& -D\left[t+L_{j}-\sum_{i=2}^{j-1}\left(T_{i-1} \ominus T_{i-1} \ominus L_{i}\right), t+L_{j}\right) .
\end{aligned}
$$

As a result, the costs for the echelons $1, \ldots, j-1$ that are directly determined by $I P_{j-1}\left(t+L_{j}\right)$ are equal to

$$
\begin{aligned}
T_{j-1} G_{j-1}\left(\operatorname { m i n } \left\{S_{j-1}, y-D\left[t-\sum_{i=2}^{j}\left(T_{i-1} \ominus L_{i}\right),\right.\right.\right. \\
\left.\left.\left.t+L_{j}-\sum_{i=2}^{j-1}\left(T_{i-1} \ominus L_{i}\right)\right)\right\}\right) .
\end{aligned}
$$

Similarly,

$$
\begin{aligned}
& I P_{j-1}\left(t+L_{j}+T_{j-1}\right) \\
& =\min \left\{S_{j-1}, y-D\left[t-\sum_{i=2}^{j}\left(T_{i-1} \ominus L_{i}\right),\right.\right. \\
& \left.\left.t+L_{j}+T_{j-1}-\sum_{i=2}^{j-1}\left(T_{i-1} \ominus L_{i}\right)\right)\right\} \\
& -D\left[t+L_{j}+T_{j-1}-\sum_{i=2}^{j-1}\left(T_{i-1} \ominus L_{i}\right), t+L_{j}+T_{j-1}\right),
\end{aligned}
$$

and the costs for the echelons $1, \ldots, j-1$ that are directly determined by $I P_{j-1}\left(t+L_{j}+T_{j-1}\right)$ are equal to

$$
\begin{aligned}
T_{j-1} G_{j-1}\left(\operatorname { m i n } \left\{S_{j-1}, y-D\left[t-\sum_{i=2}^{j}\left(T_{i-1} \ominus L_{i}\right),\right.\right.\right. \\
\left.\left.\left.t+L_{j}+T_{j-1}-\sum_{i=2}^{j-1}\left(T_{i-1} \ominus L_{i}\right)\right)\right\}\right),
\end{aligned}
$$

and so on. Taking all costs together gives

$$
\begin{aligned}
& G_{j}(y) \\
& =\frac{1}{T_{j}} \mathrm{E}\left[\sum_{l=0}^{T_{j}-1} h_{j}\left(y-D\left[t-\sum_{i=2}^{j}\left(T_{i-1} \ominus L_{i}\right), t+L_{j}+l\right]\right)\right. \\
& +T_{j-1} G_{j-1}\left(\operatorname { m i n } \left\{S_{j-1}, y-D\left[t-\sum_{i=2}^{j}\left(T_{i-1} \ominus L_{i}\right),\right.\right.\right. \\
& \left.\left.\left.t+L_{j}-\sum_{i=2}^{j-1}\left(T_{i-1} \ominus L_{i}\right)\right)\right\}\right) \\
& +T_{j-1} G_{j-1}\left(\operatorname { m i n } \left\{S_{j-1}, y-D\left[t-\sum_{i=2}^{j}\left(T_{i-1} \ominus L_{i}\right),\right.\right.\right. \\
& \left.\left.\left.\left.t+L_{j}+T_{j-1}-\sum_{i=2}^{j-1}\left(T_{i-1} \ominus L_{i}\right)\right)\right\}\right)+\cdots\right] \\
& =\frac{1}{T_{j}} \mathrm{E}\left[\sum_{l=0}^{T_{j}-1} h_{j}\left(y-D\left(L_{j}+l+1+\sum_{i=2}^{j}\left(T_{i-1} \ominus L_{i}\right)\right)\right)\right. \\
& +T_{j-1} G_{j-1}\left(\min \left\{S_{j-1}, y-D\left(L_{j}+\left(T_{j-1} \ominus L_{j}\right)\right)\right\}\right) \\
& \left.+T_{j-1} G_{j-1}\left(\min \left\{S_{j-1}, y-D\left(L_{j}+\left(T_{j-1} \ominus L_{j}\right)+T_{j-1}\right)\right\}\right)+\cdots\right] \\
& =\frac{1}{T_{j}} \sum_{l=0}^{T_{j}-1} \mathrm{E}\left[h_{j}\left(y-D\left(L_{j}+l+1+\sum_{i=2}^{j}\left(T_{i-1} \ominus L_{i}\right)\right)\right)\right. \\
& +G_{j-1}\left(\operatorname { m i n } \left\{S_{j-1}, y-D\left(L_{j}+\left(T_{j-1} \ominus L_{j}\right)\right.\right.\right. \\
& \left.\left.\left.\left.+\left\lfloor\frac{l}{T_{j-1}}\right\rfloor T_{j-1}\right)\right\}\right)\right\rfloor,
\end{aligned}
$$

which is equivalent to formula (11) for $G_{j}(y)$. This completes the proof.

Proposition 6. In this proof, we use ' (prime) to denote the derivative of a function and $a \wedge b$ to represent $\min \{a, b\}$. Also, because $\mathbf{T}$ is fixed, we omit $\mathbf{T}_{\mathbf{j}}$ in the cost functions.

For part (1), it suffices to show that $\left(G_{j}(y)\right)^{\prime} \leq\left(G_{j}^{e}(y)\right)^{\prime}$ due to their convexity. We prove this by induction on $j$. It should be noted that throughout this proof, the corresponding $S_{i}$ and $S_{i}^{e}$ are the optimal base-stock levels for $i<j$ in $G_{j}(y)$ and $G_{j}^{e}(y)$. It is trivially true for $j=1$ as $G_{1}^{e}(y)=G_{1}(y)$. Suppose it is true for $j=i-1$, i.e., $\left(G_{i-1}(y)\right)^{\prime} \leq\left(G_{i-1}^{e}(y)\right)^{\prime}$, which implies 
$S_{i-1} \geq S_{i-1}^{e}$. Then for $j=i$, let $D_{i}$ denote $D\left(L_{i}+\left\lfloor l / T_{i-1}\right\rfloor T_{i-1}\right)$, and

$$
\begin{aligned}
\left(G_{i}(y)\right)^{\prime} & =\frac{1}{T_{i}} \sum_{l=0}^{T_{i}-1} \mathrm{E}\left[h_{i}+\left(G_{i-1}\left(S_{i-1} \wedge\left(y-D\left(T_{i-1} \ominus L_{i}\right)-D_{i}\right)\right)\right)^{\prime}\right] \\
& \leq \frac{1}{T_{i}} \sum_{l=0}^{T_{i}-1} \mathrm{E}\left[h_{i}+\left(G_{i-1}\left(S_{i-1}^{e} \wedge\left(y-D\left(T_{i-1} \ominus L_{i}\right)-D_{i}\right)\right)\right)^{\prime}\right] \\
& \leq \frac{1}{T_{i}} \sum_{l=0}^{T_{i}-1} \mathrm{E}\left[h_{i}+\left(G_{i-1}^{e}\left(S_{i-1}^{e} \wedge\left(y-D\left(T_{i-1} \ominus L_{i}\right)-D_{i}\right)\right)\right)^{\prime}\right] \\
& \leq \frac{1}{T_{i}} \sum_{l=0}^{T_{i}-1} \mathrm{E}\left[h_{i}+\left(G_{i-1}^{e}\left(S_{i-1}^{e} \wedge\left(y-D_{i}\right)\right)\right)^{\prime}\right] \\
& =\left(G_{i}^{e}(y)\right)^{\prime},
\end{aligned}
$$

where the first inequality follows from that $S_{i-1}^{e} \leq S_{i-1}$ and $\left(G_{i-1}(y)\right)^{\prime} \leq 0$ for $y<S_{i-1}$, the second inequality follows from the inductive assumption, and the last inequality follows from the convexity of $G_{i-1}^{e}(y)$.

For part (2), because $\mathbf{T}$ is fixed, it is sufficient to show $G_{N}\left(S_{N}\right) \geq G_{N}^{e}\left(S_{N}^{e}\right)$. To show this, it is sufficient to show that, for any $j$,

$$
G_{j}(y) \geq E\left[G_{j}^{e}\left(y-D\left(\sum_{i=2}^{j} T_{i-1} \ominus L_{i}\right)\right)\right] \geq G_{j}^{e}\left(S_{j}^{e}\right),
$$

where the last inequality is due to the optimality of $S_{j}^{e}$. Thus we only need to prove the first inequality. We show this result by induction. It is clearly true for $j=1$ as $D\left(\sum_{i=2}^{j} T_{i-1}-L_{i}\right)=0$ and $G_{1}^{e}(y)=G_{1}(y)$. Suppose it is true for $j=i-1$. Then for $j=i$,

$G_{i}(y)$

$$
\begin{array}{r}
=\frac{1}{T_{i}} \sum_{l=0}^{T_{i}-1} \mathrm{E}\left[h_{i}\left(y-D\left(\sum_{k=2}^{i} T_{k-1} \ominus L_{k}\right)-D\left(L_{i}+l+1\right)\right)\right. \\
\left.+G_{i-1}\left(S_{i-1} \wedge\left(y-D\left(T_{i-1} \ominus L_{i}\right)-D_{i}\right)\right)\right] \\
\geq \frac{1}{T_{i}} \sum_{l=0}^{T_{i}-1} \mathrm{E}\left[h_{i}\left(y-D\left(\sum_{k=2}^{i} T_{k-1} \ominus L_{k}\right)-D\left(L_{i}+l+1\right)\right)\right. \\
+\mathrm{E}\left[G _ { i - 1 } ^ { e } \left(\left(S_{i-1} \wedge\left(y-D\left(T_{i-1} \ominus L_{i}\right)-D_{i}\right)\right)\right.\right. \\
\left.-\frac{1}{T_{i}} \sum_{l=0}^{T_{i}-1} \mathrm{E}\left[h_{i}\left(y-D\left(\sum_{k=2}^{i} T_{k-1} \ominus L_{k=2}^{i-1} T_{k-1} \ominus L_{k}\right)\right)\right]\right] \\
+\mathrm{E}\left[G _ { i - 1 } ^ { e } \left(S_{i-1}-D\left(\sum_{k=2}^{i-1} T_{k-1} \ominus L_{k}\right)\right.\right. \\
\left.\left.\wedge\left(y-D\left(\sum_{k=2}^{i} T_{k-1}-L_{k}\right)-D_{i}\right)\right)\right]
\end{array}
$$

$$
\begin{aligned}
& \geq \frac{1}{T_{i}} \sum_{l=0}^{T_{i}-1} \mathrm{E}\left[h_{i}\left(y-D\left(\sum_{k=2}^{i} T_{i-1} \ominus L_{i}\right)-D\left(L_{i}+l+1\right)\right)\right. \\
& \left.+\mathrm{E}\left[G_{i-1}^{e}\left(S_{i-1}^{e} \wedge\left(y-D\left(\sum_{k=2}^{i} T_{k-1} \ominus L_{k}\right)-D_{i}\right)\right)\right]\right] \\
& =\mathrm{E}\left[G_{i}^{e}\left(y-D\left(\sum_{k=2}^{i} T_{k-1} \ominus L_{k}\right)\right)\right],
\end{aligned}
$$

where the first equality follows from the definition of $G_{i}(y)$; the first inequality is due to the inductive assumption, the second inequality is due to the optimality of $S_{i-1}^{e}$, and the last equality follows from the definition of $G_{i}^{e}(y)$. This completes the induction, and part (2) is proved. Part (3) follows immediately from part (2) because under a fixed $T$, a local and an echelon $(s, T)$ policy have the same fixed ordering cost.

\section{Appendix B. An Approach to Find Optimal Batch} Sizes for Local $(r, n Q)$ Policies

We only sketch the idea. The detailed analysis can be requested from the authors.

Let the optimal local $(r, Q)$ policy be $\left(r_{j}^{*}, Q_{j}^{*}\right)$ and the resulting optimal cost be $C\left(\mathbf{r}^{*}, \mathbf{Q}^{*}\right)$. Define

$$
\begin{aligned}
C^{e}(\mathbf{Q})= & \text { the total cost obtained from an echelon }(r, Q) \\
& \text { policy with batch sizes } \mathbf{Q} \text {, and the } \\
& \text { corresponding best echelon reorder points } \\
& \mathbf{R}(\mathbf{Q})=\left(R_{1}\left(Q_{1}\right), \ldots, R_{N}\left(\mathbf{Q}_{\mathbf{N}}\right)\right), \\
G_{j}^{e}\left(\mathbf{Q}_{\mathbf{j}}\right)= & \text { the cost for the echelon stage } j \text { with batch } \\
& \text { sizes } \mathbf{Q}_{\mathbf{j}}=\left(Q_{1}, \ldots, Q_{j}\right) \text { and the } \\
& \text { corresponding best reorder points } \\
& \mathbf{R}_{\mathbf{j}}\left(\mathbf{Q}_{\mathbf{j}}\right)=\left(R_{1}\left(Q_{1}\right), \ldots, R_{j}\left(\mathbf{Q}_{\mathbf{j}}\right)\right) \\
\underline{c}_{j}\left(Q_{j}\right)= & \text { a lower bound to the cost for stage } j \text { with } \\
& \text { batch size } Q_{j}, \\
G_{j}^{l}\left(Q_{j}\right)= & \text { a lower bound to } G_{j}^{e}\left(\mathbf{Q}_{\mathbf{j}}\right) .
\end{aligned}
$$

We briefly explain how to obtain the above cost functions from the literature. For fixed $\mathbf{Q}$, the best echelon reorder points $\mathbf{R}(\mathbf{Q})$ and the optimal cost $C^{e}(\mathbf{Q})$ can be obtained from Chen (2000). The echelon cost function $G_{j}^{e}\left(\mathbf{Q}_{j}\right)$ and the lower bound $G_{j}^{l}(Q)$ are derived in Shang and Song (2007). The lower bound $\underline{c}_{j}(Q)$ to the stage cost is derived in Shang and Zhou (2009).

Let $C^{h}$ be a heuristic cost for the local $(r, Q)$ policy. It can be shown that

$$
\begin{aligned}
C^{h} \geq C\left(\mathbf{r}^{*}, \mathbf{Q}^{*}\right) & \geq C^{e}\left(\mathbf{Q}^{*}\right)>G_{N}^{e}\left(Q_{N}^{*}\right) \\
& \geq \sum_{i=j+1}^{N} \underline{c}_{i}\left(Q_{i}^{*}\right)+G_{j}^{l}\left(Q_{j}^{*}\right)+\pi_{j} .
\end{aligned}
$$

The second inequality in (15) follows from the fact that a local policy is a special case of the echelon $(r, Q)$ policy (Axsäter and Rosling 1993). The rest of the inequality is from Shang and Zhou (2009).

Note that (15) is similar to (12). Thus we can find the bounds for $Q_{j}^{*}$ by using the same procedure as the one 
for finding the bounds for local $T_{j}^{*}$. That is, starting from stage $N$, we can find the bounds $\left[\underline{Q}_{N}, \bar{Q}_{N}\right]$ for $Q_{N}^{*}$. Then for $Q_{N} \in\left[\underline{Q}_{N}, \bar{Q}_{N}\right]$, we search for the minimum $\underline{c}_{N}\left(Q_{N}\right)$, referred to as $\underline{\underline{c}}_{N}$. Next, applying (15) again by setting $j=N-1$, we find the solution bounds for $Q_{N-1}^{*}$ and then $\underline{c}_{N-1}$, etc., until $j=1$. After the solution bounds are established, one can apply the algorithm in Chen (1998b) to search for the optimal local reorder points for each feasible solution. The optimal local $(r, n Q)$ policy can be found by evaluating all feasible solutions and their corresponding optimal local reorder points. (One can evaluate a local $(r, n Q)$ policy by using the evaluation scheme for the echelon $(r, n Q)$ policy because the former is a special case of the latter; see Chen 1998b.)

\section{References}

Aviv, Y., A. Federgruen. 1998. The operational benefits of information sharing and vendor managed inventory (VMI) programs. Working paper, Washington University in St. Louis, St. Louis.

Axsäter, S. 1993. Exact and approximate evaluation of batchordering policies for two-level inventory systems. Oper. Res. 41 777-785.

Axsäter, S. 2003. Supply chain operations: Serial and distribution inventory systems. A. G. de Kok, S. C. Graves, eds. Supply Chain Management: Design, Coordination, and Operation, Vol. 11. Handbooks in Operations Research and Management Science. Elsevier, Amsterdam, 525-559.

Axsäter, S., L. Juntti. 1996. Comparison of echelon stock and installation stock policies for two-level inventory systems. Internat. J. Production Econom. 45 303-310.

Axsäter, S., K. Rosling. 1993. Installation vs. echelon stock policies for multi-level inventory control. Management Sci. 39 1274-1280.

Cachon, G. 1999. Managing supply chain demand variability with scheduled ordering policies. Management Sci. 45 $843-856$.

Cachon, G. 2001a. Exact evaluation of batch-ordering policies in two-echelon supply chains with periodic review. Oper. Res. 49 79-98.

Cachon, G. 2001b. Managing a retailer's shelf space, inventory, and transportation. Manufacturing Service Oper. Management 3 211-229.

Cachon, G., M. Fisher. 2000. Supply chain inventory management and the value of shared information. Management Sci. 46 1032-1048.

Chao, X., S. Zhou. 2009. Optimal policy for multi-echelon inventory system with batch ordering and fixed replenishment intervals. Oper. Res. 57 377-390.

Chen, F. 1998a. On $(R, n Q)$ policies in serial inventory systems. S. Tayur, R. Ganeshar, M. Magazine, eds. Quantitative Models for Supply Chain Management. Kluwer Academic Publishers, Norwell, MA, 71-109.
Chen, F. 1998b. Echelon reorder points, installation reorder points, and the value of centralized demand information. Management Sci. 44 S221-S234.

Chen, F. 2000. Optimal policies for multi-echelon inventory problems with batch ordering. Oper. Res. 48 376-389.

Chen, F., Y. S. Zheng. 1998. Near-optimal echelon-stock $(r, n q)$ policies in multi-stage serial systems. Oper. Res. 46 592-602.

De Bodt, M., S. Graves. 1985. Continuous review policies for a multi-echelon inventory problem with stochastic demand. Management Sci. 31 1286-1295.

Feng, K., U. S. Rao. 2007. Echelon-stock $(R, n T)$ control in two-stage serial stochastic inventory systems. Oper. Res. Lett. 35 95-104.

Gavirneni, S. 2002. Information flows in capacitated supply chains with fixed ordering costs. Management Sci. 48 644-651.

Gavirneni, S., R. Kapuscinski, S. Tayur. 1999. Value of information in capacitated supply chains. Management Sci. 45 16-24.

Graves, S. 1996. A multiechelon inventory model with fixed replenishment intervals. Management Sci. 42 1-18.

Graves, S. 1999. A single-item inventory model for a non-stationary demand process. Manufacturing Service Oper. Management 1 $50-61$.

Gürbüz, M., K. Moinzadeh, Y.-P. Zhou. 2007. Coordinated replenishment strategies in inventory/distribution systems. Management Sci. 53 293-307.

Hadley, G., T. Whitin. 1963. Analysis of Inventory Systems, PrenticeHall, Englewood Cliffs, NJ.

Lee, H., K. So, C. Tang. 2000. The value of information sharing in two-level supply chain. Management Sci. 46 626-643.

Naddor, E. 1975. Optimal and heuristic decisions in single- and multi-item inventory systems. Management Sci. 21 1234-1249.

Rao, U. S. 2003. Properties of the period review $(R, T)$ inventory control policy for stationary, stochastic demand. Manufacturing Service Oper. Management 5 37-53.

Shang, K. 2008. Note: A simple heuristic for serial inventory systems with fixed order costs. Oper. Res. 56 1039-1043.

Shang, K., J. Song. 2007. Supply chains with economies of scale: Single-stage heuristic and approximations. Oper. Res. 55 843-853.

Shang, K., S. Zhou. 2009. Optimal and heuristic $(r, n Q, T)$ policies in serial inventory systems with fixed costs. Oper. Res. Forthcoming.

Shang, K., J. Song, P. Zipkin. 2009. Coordination mechanisms in decentralized serial inventory systems with batch ordering. Management Sci. 55 685-695.

Simchi-Levi, D., Y. Zhao. 2004. The value of information sharing in a two-stage supply chain with production capacity constraints. Probab. Engrg. Inform. Sci. 18 247-274.

Simchi-Levi, D., Y. Zhao. 2007. Three generic methods for evaluating stochastic multi-echelon inventory systems. Working paper, Massachusetts Institute of Technology, Cambridge.

van Houtum, G. J., A. Scheller-Wolf, J. Yi. 2007. Optimal control of serial, multi-echelon inventory/production systems with fixed replenishment intervals. Oper. Res. 55 674-687.

Willems, S. 2008. Data set-real-world multiechelon supply chains used for inventory optimization. Manufacturing Service Oper. Management 10 19-23. 